
but does not induce genomic and physiological changes relevant for human health Moissl-Eichinger ${ }^{1,7,+}$

${ }^{1}$ Medical University of Graz, Department of Internal Medicine, Graz, Austria Research Group Astrobiology, Cologne, Germany

${ }^{3}$ University of Edinburgh, School of Physics and Astronomy, Edinburgh, UK

${ }^{4}$ European Space Research and Technology Centre (ESTEC), Noordwijk, The Netherlands

${ }^{5}$ Lomonosov Moscow State University, Biological Faculty, Moscow, Russia

${ }^{6}$ Ludwig Maximilians University of Munich, Plant Development and Electron Microscopy, Department of Biology I, Biocenter, Planegg-Martinsried, Germany

${ }^{7}$ BioTechMed Graz, Austria

${ }^{+}$Corresponding author. christine.moissl-eichinger@medunigraz.at

The International Space Station selects for microorganisms adapted to the extreme environment,

Maximilian Mora ${ }^{1}$, Lisa Wink ${ }^{1}$, Ines Kögler ${ }^{1}$, Alexander Mahnert ${ }^{1}$, Petra Rettberg ${ }^{2}$, Petra Schwendner ${ }^{3}$, René Demets ${ }^{4}$, Charles Cockell $^{3}$, Tatiana Alekhova ${ }^{5}$, Andreas Klingl ${ }^{6}$, Alina Alexandrova ${ }^{5}$, Christine

${ }^{2}$ German Aerospace Center (DLR), Institute of Aerospace Medicine, Radiation Biology Department, 
The International Space Station (ISS) is a unique, completely confined habitat for the human crew and co-inhabiting microorganisms. Here, we report on the results of the ISS experiment "EXTREMOPHILES". We aimed to exploit the microbial information obtained from three surface and air sampling events aboard the International Space Station during increments 51 and 52 (2017) with respect to: i) microbial sources, diversity and distribution within the ISS, ii) functional capacity of microbiome and microbial isolates, iii) extremotolerance and antibiotics-resistance (compared to ground controls), and iv) microbial behavior towards ISS-relevant materials such as biofilm formation, or potential for degradation. We used wipe samples and analyzed them by amplicon and metagenomics sequencing, cultivation, comparative physiological studies, antibiotic resistance tests, genome analysis of isolates and co-incubation experiments with ISS-relevant materials. The major findings were: i) the ISS microbiome profile is highly similar to ground-based confined indoor environments, ii) the ISS microbiome is subject to fluctuations and indicative for the (functional) location, although a core microbiome was present over time and independent from location, iii) the ISS selects for microorganisms adapted to the extreme environment, but does not necessarily induce genomic and physiological changes which might be relevant for human health, iv) cleanrooms and cargo seems to be a minor source of microbial contamination aboard, and v) microorganisms can attach to and grow on ISS-relevant materials. Biofilm formation might be a threat for spacecraft materials with the potential to induce instrument malfunctioning with consequences for mission success. We conclude that our data do not raise direct reason for concern with respect to crew health, but indicate a potential threat towards biofilm formation and material integrity in moist areas. 
Introduction

Human space exploration beyond boundaries of Earth and Moon is a declared goal of NASA, ESA, Roscosmos and other space-faring agencies, envisaging a potential human Mars mission in the next 20 to 30 years. Maintenance of astronauts' health during a several hundred days journey in a confined artificial environment in space is one of the key aspects which has to be addressed for such a long-term mission.

The human immune system was shown to be compromised under space flight conditions, as a significant decrease of lymphocytes and also of the activity of innate and adaptive immune response was observed when compared to terrestrial controls $(1,2)$. Adding an order of complexity, human health is strongly intertwined with its microbiome, billions of microorganisms thriving on external and internal surfaces of the human body.

Our body's microbiome is dependent on the environmental microbiome, as they are in constant exchange and interaction. Isolated human subjects in hospitals were observed to loose microbial diversity, serving as an indicator for health and stability in general (Koskinen 2019, unpublished); this might, however, be contradictory to observations on the astronauts' gut microbiome (3). The microbiome is prone to external factors, which influence the composition or the function of the microbial community. For instance, microbial diversity in built environments was shown to negatively correlate with the diversity of antimicrobial resistances especially in confined habitats in a recent publication (Mahnert et al, 2019, unpublished). With respect to space travel, it has been shown that microgravity affects the virulence of certain microorganisms, such as Salmonella typhimurium (4), Listeria monocytogenes and Enterococcus faecalis (5). As a consequence, monitoring of the microbial community aboard spacecraft is highly important to assess risk factors to the health of crew members.

Additionally, some microorganisms might even pose a risk to the material integrity of a spacecraft: So-called technophilic microorganisms, in particular fungi, are able to corrode alloys and polymers used in spacecraft assembly (6). Technophilic microorganisms caused major problems on the former Russian space station Mir $(7,8)$.

The majority of information with respect to environmental microbiome dynamics aboard manned spacecraft is retrieved from ground-based simulation studies, such as the Mars500 (9) and the HISEAS (http://hi-seas.org/) experiments. However, the ISS is currently representing the most isolated human habitat. 
The ISS circles our planet in low Earth orbit (approx. $400 \mathrm{~km}$ above ground) and is constantly inhabited since more than 18 years. Except for cargo exchange and the arrival of new crew members roughly every six months, the ISS is completely sealed off from any surrounding biological ecosystem and thus represents one of the most isolated and confined man-made environments to date (10). Like no other currently available testbed for long-term manned space missions, the ISS has the scientific benefit of providing real spaceflight conditions, including microgravity and an elevated background radiation - parameters, which are hard to implement in ground-based simulation studies.

The ISS consists of different modules, and while new modules were added over the years, also the crew size increased. Nowadays six international astronauts and cosmonauts routinely inhabit the ISS, along with their associated microorganisms. While certain microorganisms might benefit from the constant temperature (approx. $22^{\circ} \mathrm{C}$ ) and stable humidity (approx. 60\%) aboard the ISS, aforementioned harsh spaceflight conditions as well as low nutrient levels due to regular cleaning and a reduced introduction of new material, make the ISS for microorganisms a unique and extremesituated indoor environment (11).

Recent publications focused on the microbial analysis of ISS debris and dust (12-15), the study of the astronauts' microbiome (16), the characterization of bacterial and fungal isolates from the ISS $(17,18)$ and the (molecular) microbial analysis of swab and wipe samples taken inside the ISS (19). A study investigating the growth behavior of non-pathogenic (terrestrial) bacteria aboard the ISS found no changes in most bacteria, given that they have enough nutrients (11).

Other publications also focused on the detection of antimicrobial resistance genes aboard the ISS and evaluated the potential risk these genes might represent in a closed spacecraft environment (20). Singh et al. assessed the succession and persistence of microbial communities and the associated antimicrobial resistance and virulence properties based on metagenomic reads obtained from samples of three flights. Overall, 46 microbial species were found, including eight biorisk group 2 species, to be persistent on the ISS over a timespan of roughly one and a half years (21). The authors inferred an increase of antimicrobial resistance and virulence genes over time bearing an alarming message that these factors seem to become an increasing proportion of the ISS microbiome.

Although an increased risk for the health of the astronauts and cosmonauts aboard has been proposed several times based on the molecular detection of antimicrobial resistances, viruses and pathogens, infections of crew members or health issues related to pathogenic action of microorganisms have been reported only rarely (22). Moreover, the given environmental microbial contamination limits (air, surfaces) were exceeded only in a few cases to date, in which appropriate 
117 countermeasures succeeded in a timely manner (23). Moreover, a recent genomics-based study 118 could not reveal potentially health-threatening differences in Bacillus and Staphylococcus 119 pangenomes from ISS, compared to human-associated and soil pangenomes (24).

120 In this study, we report on the realization of the ISS experiment EXTREMOPHILES, targeting the 121 profile, diversity, dynamics and functional capacity of the microbiome aboard. We used cultivation 122 efforts to obtain microbial isolates. We assessed their genomic and physiological adaptation towards 123 ISS conditions and tested the hypothesis, whether, as indicated by previous literature reports, ISS 124 microorganisms possess a higher extremo-tolerance and antibiotics-resistance potential compared 125 toground controls. Moreover, we were interested in the surface-microbe interaction with regard to 126 material integrity, exhibited by selected, freshly-isolated ISS strains. 
Pre-flight preparations and sampling aboard the ISS. Packaging, pre-processing and logistics of the sample material regarding upload and download from the ISS were managed by the Biotechnology Space Support Center (BIOTESC) of the Lucerne University of Applied Sciences and Arts (Switzerland). In-flight sampling aboard the ISS was performed during increment 51 and 52 (April to June 2017) under the ESA (European Space Agency) operation named EXTREMOPHILES. Sampling was performed either with dry wipes (session A and B) or pre-moistened wipes (session C; TX 3211 Alpha Wipe, ITW Texwipe, Kernersville, US, $23 \times 23 \mathrm{~cm} ; 20 \mathrm{ml}$ autoclaved ultra-pure water for chromatography, LiChrosolv, Merck Millipore). Three sampling sessions were performed, with session A on 01.05.2017, session $B$ on 12.07.2017 and session $C$ on 29.06.2017. With a span of 72 days between session $A$ and $B$ they were conceptualized for comparative sampling to assess microbiome fluctuation over time. An overview of all sampled areas and sessions is given in Table 1. different locations within the ISS.

\begin{tabular}{|c|c|c|c|}
\hline Wipe & Sampled surface & ISS module & Session \\
\hline A-5, B-1 & Ambient air (field blank, FB) & \multirow[t]{5}{*}{ Columbus } & \multirow[t]{8}{*}{$A, B$} \\
\hline A-4, B-2 & Light covers & & \\
\hline A-2, B-3 & SSC laptop & & \\
\hline A-3, B-4 & Hand grips & & \\
\hline A-1, B-5 & Return grid sensor housing (RGSH) & & \\
\hline A-6, B-6 & Sleeping Unit & \multirow{4}{*}{$\begin{array}{l}\text { Node } 2 \\
\text { (Harmony) }\end{array}$} & \\
\hline A-7, B-7 & $\begin{array}{l}\text { Panels (outer surface, close to the portable fire } \\
\text { extinguisher (PFA) and portable breathing apparatus } \\
\text { (PBA)) }\end{array}$ & & \\
\hline$A-8, B-8$ & Audio terminal unit (ATU) & & \\
\hline A-9 & Return Grid Sensor Housing (RGSH) & & A \\
\hline $\mathrm{C}-1$ & Ambient air (field blank, FB) & \multirow[t]{2}{*}{ Cupola } & \multirow[t]{7}{*}{$\mathrm{C}$} \\
\hline $\mathrm{C}-2$ & Surface facing a window & & \\
\hline $\mathrm{C}-3$ & Advanced resistive exercise device (ARED) & \multirow{3}{*}{$\begin{array}{l}\text { Node } 3 \\
\text { (Tranquility) }\end{array}$} & \\
\hline C-4 & Treadmill & & \\
\hline $\mathrm{C}-5$ & Waste and hygiene compartment (WHC): surfaces & & \\
\hline C-6 & Cover of the PBA, inside & \multirow[t]{2}{*}{ Node 1 (Unity) } & \\
\hline$C-7$ & Dining table & & \\
\hline
\end{tabular}

The sampling instructions for each session were as follows. 1: Put on sterile gloves (DNA-free nitrile gloves, ABF Diagnostics GmbH, Kranzberg, Germany). 2: Using gloved hand, remove wipe X from bag (metal closure bag, GML-alfaplast $\mathrm{GmbH}$, Munich, Germany), wave wipe through the air (approx. 20s). Put wipe back into its bag and close properly. 3: Change glove. 4: Using gloved hand, take sample using wipe $Y$ according to Table 1. Put wipe back into its bag and close properly. 5: Repeat 
steps 3 and 4 for every new sampling surface according to Table 1. 6: Store wipes at ambient (session A, B, dry wipes), or under cool conditions ("cold stowage", 2-10 $\mathrm{C}$, session $\mathrm{C}$, moist wipes).

Cleanroom and cargo vehicle sampling. In order to retrieve samples for comparative analyses, one ISS-relevant cleanroom and cargo-spacecraft was sampled, namely cleanroom S5C at the Centre Spatial Guayanais near Kourou in French Guiana, housing ATV5 "Georges Lemaître". Swab (FLOQSwabs ${ }^{\mathrm{TM}}$, Copan diagnostics, USA) and wipe samples from ATV and its cleanroom were provided by Stefanie Raffestin (ESA) in 2014.

Sample extraction. The obtained sample material was either available as wipes or swabs (cleanroom). Wipes were submerged in $80 \mathrm{ml}$ DNA-free $0.9 \%(\mathrm{w} / \mathrm{v}) \mathrm{NaCl}$ solution $(\mathrm{NaCl}$ was heattreated to destroy DNA residues for 24 hours, $250^{\circ} \mathrm{C}$ ), vortexed (10s) and shaken manually (15s), ultra-sonicated at $40 \mathrm{kHz}$ for $2 \mathrm{~min}$ and vortexed (10s). The sampling material was aseptically removed from the extraction solution before cultivation- and molecular analyses. Swabs were submerged in $15 \mathrm{ml}$ of $\mathrm{NaCl}$ solution and processed identically.

Cultivation. Cultivation of microorganisms was performed on a number of solid and liquid media, as given in Table 2. For microbial enrichment, we provided variable chemical and physical conditions with respect to: $\mathrm{pH}(\mathrm{pH} 4-10)$, temperature $\left(4-65^{\circ} \mathrm{C}\right.$ ), gas phase (aerobic, $\mathrm{N}_{2}: \mathrm{H}_{2}: \mathrm{CO}_{2}, \mathrm{H}_{2}: \mathrm{CO}_{2}, \mathrm{~N}_{2}: \mathrm{CO}_{2}$ ), nutrients and nutrient availability. R2A (pH5-7), RAVAN and ROGOSA were supplemented with nystatin $(50 \mu \mathrm{g} / \mathrm{ml})$ to suppress growth of fungi; media targeting archaea were supplemented with 50 $\mu \mathrm{g} / \mathrm{ml}$ streptomycin and $100 \mu \mathrm{g} / \mathrm{ml}$ ampicillin. Inoculation was done using 500 and $250 \mu \mathrm{l}$ (duplicates) of the extraction solution. In addition, $500 \mu \mathrm{l}$ aliquots of the wipe suspension were irradiated at the DLR in Cologne, Germany, to select for radiation resistant isolates. They were either irradiated by UVC $(254 \mathrm{~nm})$ with an intensity of $50 \mathrm{~J} / \mathrm{m}^{2}, 75 \mathrm{~J} / \mathrm{m}^{2}, 100 \mathrm{~J} / \mathrm{m}^{2}$, and $200 \mathrm{~J} / \mathrm{m}^{2}$ or by X-Rays with an intensity of $125 \mathrm{~Gy}, 250 \mathrm{~Gy}, 500 \mathrm{~Gy}, 750 \mathrm{~Gy}$, or $1000 \mathrm{~Gy}$. Radiation resistant microorganisms were cultivated on R2A and TSA agar. Pure cultures were obtained via repeated dilution series in liquid medium and/or purification streaks on solid media. 


\begin{tabular}{|c|c|c|c|c|c|c|c|}
\hline Medium & Abb. & Target organisms & $\mathrm{T}\left[{ }^{\circ} \mathrm{C}\right]$ & $\mathrm{pH}$ & Phase & Gas phase & $\begin{array}{l}\text { Reference or } \\
\text { manufacturer }\end{array}$ \\
\hline $\mathrm{R} 2 \mathrm{~A} \mathrm{pH} 4$ & R4 & Acidophiles & 32 & 4 & Solid & Aerobic (ambient) & \multirow{7}{*}{$\begin{array}{l}\text { VWR Chemicals } \\
\text { BDH Prolabo }^{\circledR}\end{array}$} \\
\hline R2A pH 5 & R5 & Acidotolerants & 32 & 5 & Solid & Aerobic (ambient) & \\
\hline \multirow[t]{3}{*}{$\mathrm{R} 2 \mathrm{~A} \mathrm{pH} 7$} & R7W & Mesophiles & 32 & 7 & Solid & Aerobic (ambient) & \\
\hline & R7K & Psychrophiles & 4 & 7 & Solid & Aerobic (ambient) & \\
\hline & R7A & Anaerobes & 32 & 7 & Solid & $\mathrm{N}_{2:} \mathrm{H}_{2:} \mathrm{CO}_{2}(80: 10: 10)$ & \\
\hline $\mathrm{R} 2 \mathrm{~A} \mathrm{pH} 9$ & R9 & Alkalitolerants & 32 & 9 & Solid & Aerobic (ambient) & \\
\hline $\mathrm{R} 2 \mathrm{~A} \mathrm{pH} 10$ & R10 & Alkaliphiles & 32 & 10 & Solid & Aerobic (ambient) & \\
\hline RAVAN & RAV & Oligotrophs & 32 & 7 & Solid & Aerobic (ambient) & $\begin{array}{l}(25)(1: 100 \\
\text { modified })\end{array}$ \\
\hline Halo medium & HLO & Halophiles & 40 & 7.5 & Solid & Aerobic (ambient) & $\begin{array}{l}\text { DSMZ medium } 97 \\
\text { (https://www.dsmz } \\
. \text { de/) }\end{array}$ \\
\hline Rogosa & ROG & Lactobacilli & 32 & 5.5 & Solid & Aerobic (ambient) & Merck \\
\hline Tryptic Soy Agar & TSA & Mesophiles & 32 & & Solid & Aerobic (ambient) & Becton Dickinson \\
\hline $\begin{array}{l}\text { Yeast-extract } \\
\text { peptone medium }\end{array}$ & YPD & Yeasts \& Fungi & 32 & 7 & Solid & Aerobic (ambient) & Sigma-Aldrich \\
\hline Potato dextrose agar & PDA & Yeasts \& Fungi & 25 & 5.6 & Solid & Aerobic (ambient) & Sigma-Aldrich \\
\hline $\begin{array}{l}\text { Autotrophic } \\
\text { homoacetogen } \\
\text { medium }\end{array}$ & $\mathrm{AHM}$ & Homoacetogen & 32 & 7 & Liquid & $\mathrm{H}_{2:} \mathrm{CO}_{2}(20: 80)$ & (26) \\
\hline $\begin{array}{l}\text { Autotrophic } \\
\text { allrounder medium }\end{array}$ & AAM & Autotrophs & 32 & 7 & Liquid & $\mathrm{N}_{2:} \mathrm{CO}_{2}(80: 20)$ & (26) \\
\hline $\begin{array}{l}\text { Archaea supporting } \\
\text { medium }\end{array}$ & ASM & Archaea & 32 & 7 & Liquid & $\mathrm{H}_{2:} \mathrm{CO}_{2}(20: 80)$ & (26) \\
\hline $\begin{array}{l}\text { Medium for } \\
\text { methanogens }\end{array}$ & MS & Methanogens & 40 & 7 & Liquid & $\mathrm{H}_{2:} \mathrm{CO}_{2}(20: 80)$ & (27) \\
\hline MS+organics & MSO & Methanogens & 40 & 7 & Liquid & $\mathrm{H}_{2:} \mathrm{CO}_{2}(20: 80)$ & This study \\
\hline N. exaquare medium & NEX & Thaumarchaea & 32 & 8.5 & Liquid & Aerobic (ambient) & $(28)$ \\
\hline $\begin{array}{l}\text { Mixed ruminal } \\
\text { bacteria medium }\end{array}$ & MCB-3 & Methanogens & 40 & 7 & Liquid & $\mathrm{H}_{2:} \mathrm{CO}_{2}(20: 80)$ & $\begin{array}{l}\text { DSMZ medium322 } \\
\text { (https://www.dsmz } \\
\text {.de/) }\end{array}$ \\
\hline R2A pH 7 liquid & $\mathrm{R} 2 \mathrm{~A}$ & Thermophiles & 65 & 7 & Liquid & Aerobic (ambient) & This study \\
\hline
\end{tabular}

178 16S rRNA gene sequencing and classification of the bacterial and fungal isolates. Partial 16S rRNA

179 genes of the isolates were amplified using the primers 9bF (5'-GRGTTTGATCCTGGCTCAG-3') and

180 1406uR (5'-ACGGGCGGTGTGTRCAA-3'), applying the following cycling conditions: Initial denaturation

181 at $95^{\circ} \mathrm{C}$ for $2 \mathrm{~min}$, followed by $10 \mathrm{cycles}$ of denaturing at $96^{\circ} \mathrm{C}$ for $30 \mathrm{~s}$, annealing at $60^{\circ} \mathrm{C}$ for $30 \mathrm{~s}$ and

182 elongation at $72^{\circ} \mathrm{C}$ for $60 \mathrm{~s}$, followed by another 22 cycles of denaturing at $94^{\circ} \mathrm{C}$ for $30 \mathrm{~s}$, annealing at $18360^{\circ} \mathrm{C}$ for $30 \mathrm{~s}$ and elongation at $72^{\circ} \mathrm{C}$ for $60 \mathrm{~s}$, and a final elongation step at $72^{\circ} \mathrm{C}$ for $10 \mathrm{~min}$ (29). The 184 template was either a small fraction of a picked colony in a colony-PCR assay or 5-20ng of DNA 185 purified from culture via the peqGOLD Bacterial DNA Kit (Peqlab, Germany). The 16S rRNA gene 186 amplicons were visualized on a 1.5\% agarose gel, purified with the Min Elute PCR Purification Kit 
(Qiagen, Netherlands) or the Monarch PCR and DNA Cleanup Kit (New England Biolabs, US). After Sanger-sequencing (Eurofins, Germany) the obtained sequences were classified using the EzBioCloud platform at http://www.ezbiocloud.net/eztaxon (30).

The ITS region of fungal isolates was sequenced using the primers ITS1F (5'CTTGGTCATTTAGAGGAAGTAA-3') and ITS4 (5'-TCCTCCGCTTATTGATATGC- $3^{\prime}$ ) and following cycling conditions: initial denaturation at $95^{\circ} \mathrm{C}$ for $10 \mathrm{~min}$, followed by 35 cycles of denaturing at $94^{\circ} \mathrm{C}$ for $60 \mathrm{~s}$, annealing at $51^{\circ} \mathrm{C}$ for $60 \mathrm{~s}$, elongation at $72^{\circ} \mathrm{C}$ for $60 \mathrm{~s}$, and a final elongation step at $72^{\circ} \mathrm{C}$ for $8 \mathrm{~min}$. The amplicons were Sanger-sequenced (Eurofins, Germany) and the obtained sequence was classified using the curated databases UNITE version 7.2 (31) and BOLD version 4 (32). Fungal isolates of session A, B, and C were classified according to phenotypical characteristics

Phylogenetic tree reconstruction. For phylogenetic tree reconstruction, the forward and reverse sequences obtained from the isolates were merged to reach a minimum sequence length of $1000 \mathrm{bp}$. The phylogenetic tree was calculated with the Fast Tree programme (33) and displayed with the Interactive Tree of Life online tool iTOL (34).

DNA extraction of ISS wipe samples. After aliquots were removed for cultivation assays, the rest of the wipe solutions were filled into Amicon Ultra-15 filter tubes (Sigma Aldrich) and were centrifuged at $4000 \mathrm{x}$ for $10-30 \mathrm{~min}$ at $4^{\circ} \mathrm{C}$. The flow-through was discarded and the remaining liquid in the filters was pipetted into $1.5 \mathrm{ml}$ Eppendorf tubes for DNA extraction with the modified XS- buffer method as previously described (35). DNA concentrations were determined using Qubit (Life Technologies, US).

Microbial profiling using next- generation sequencing methods. To investigate the detectable molecular diversity, we used a "universal" and an Archaea-targeting approach. The 16S rRNA gene amplicons for the universal approach were amplified using Illumina-tagged primers F515 (5'TCGTCGG-CAGCGTCAGATGTGtATAAGAGACAGGTGCCAGCMGCCGCGGTAA-3') and R806 (5'amplicons were obtained by a nested approach (37): First, a 550 bp-long 16S rRNA gene amplicon was generated with the primers Arch344F (5'-ACGGGGYGCAGCAGGCGCGA-3') and Arch915R (5'GTGCTCCCCCGCCAATTCCT-3') $(38,39)$ and in a second PCR, the amplicons for Illumina sequencing were generated by the tagged primers

Arch519F $\left(5^{\prime}-\right.$ purified product of the first PCR as template. The cycling conditions for the universal approach were initial denaturation at $94^{\circ} \mathrm{C}$ for $3 \mathrm{~min}$, followed by 35 cycles of denaturing at $94^{\circ} \mathrm{C}$ for $45 \mathrm{~s}$, annealing 
at $60^{\circ} \mathrm{C}$ for $60 \mathrm{~s}$ and elongation at $72^{\circ} \mathrm{C}$ for $90 \mathrm{~s}$, followed by a final elongation step at $72^{\circ} \mathrm{C}$ for $10 \mathrm{~min}$. For the first PCR of the nested archaeal approach, the cycling conditions were initial denaturation at $95^{\circ} \mathrm{C}$ for $2 \mathrm{~min}$, followed by $10 \mathrm{cycles}$ of denaturing at $96^{\circ} \mathrm{C}$ for $30 \mathrm{~s}$, annealing at $60^{\circ} \mathrm{C}$ for $30 \mathrm{~s}$, and elongation at $72^{\circ} \mathrm{C}$ for $60 \mathrm{~s}$, followed by another 15 cycles of denaturing at $94^{\circ} \mathrm{C}$ for $30 \mathrm{~s}$, annealing at $60^{\circ} \mathrm{C}$ for $30 \mathrm{~s}$, and elongation at $72^{\circ} \mathrm{C}$ for $60 \mathrm{~s}$, and a final elongation step at $72^{\circ} \mathrm{C}$ for $10 \mathrm{~min}$. For the second amplification the cycling conditions were initial denaturation at $95^{\circ} \mathrm{C}$ for $5 \mathrm{~min}$, followed by 25 cycles of denaturing at $95^{\circ} \mathrm{C}$ for $40 \mathrm{~s}$, annealing at $63^{\circ} \mathrm{C}$ for $120 \mathrm{~s}$ and elongation at $72^{\circ} \mathrm{C}$ for $60 \mathrm{~s}$, followed by a final elongation step at $72^{\circ} \mathrm{C}$ for $10 \mathrm{~min}$.

Genome sequencing, genome reconstruction and annotation of selected isolates. We sequenced the genomic DNA of six isolates obtained from ISS samples described earlier (41). DNA was isolated from overnight cultures using the peqGOLD bacterial DNA mini kit (Peqlab, Germany). Double stranded DNA was quantified via Qubit Fluorometer 2.0 (Invitrogen, USA) according to manufacturer's instructions. Library preparation and sequencing was carried out at the Core Facility Molecular Biology at the Center for Medical Research at the Medical University Graz, Austria.

Genomic reads were quality checked with FastQC (42) and then filtered with Trimmomatic (removed all adapter sequences, SLIDINGWINDOW 4:20, MINLEN 50)(43). Genomes were assembled with SPADES in careful mode (44) and afterwards checked for completeness via CheckM (45). The assemblies were annotated and compared to closely related reference strains via the microbial genome annotation \& analysis platform MicroScope (http://www.genoscope.cns.fr/agc/microscope) (46-48).

Resistance and physiological tests. Experiments were performed with selected microbial isolates from this and our recent study on ISS microorganisms (14). (i) Heat- shock resistance test: The heatshock test was carried out according to ESA standards (49). In brief, single colonies of 3-5-day old cultures were suspended in two test tubes containing $2.5 \mathrm{ml}$ sterile PBS. One tube was incubated at room temperature (control), whereas the other was placed in a water bath and exposed for $15 \mathrm{~min}$ to $80^{\circ} \mathrm{C}$. Samples were immediately cooled down on ice for $5 \mathrm{~min}$ after incubation time. The temperature was monitored using a separate pilot tube containing $2.5 \mathrm{ml} \mathrm{PBS}$. Afterwards, $0.5 \mathrm{ml}$ of the heat-shocked suspension and $0.5 \mathrm{ml}$ of the room temperature suspension were plated and incubated at $32^{\circ} \mathrm{C}$ for $72 \mathrm{~h}$. (ii) Physiological tests: For the assessment of the temperature range, cultures were plated on $\mathrm{R} 2 \mathrm{~A} \mathrm{pH7}$ agar and incubated overnight at $32^{\circ} \mathrm{C}$. Then the incubation temperatures for the species still growing were stepwise decreased and increased until no further growth was observed. Limits of pH tolerance were assessed accordingly. (iii) Antibiotics susceptibility tests: Antimicrobial susceptibility testing for selected, clinically relevant antibiotics (Table 3) was performed using Etest ${ }^{\circledR}$ reagent strips (Biomérieux, Germany) according to manufacturer's 
instruction and detailed in (14). Since there were no species-specific breakpoints available, MICs were interpreted according to EUCAST guideline table "PK/PD (Non-species related) breakpoints" (50). In brief, overnight cultures (2-3-day cultures for slower-growing bacteria) were suspended in 0.9\% saline. $100 \mu \mathrm{l}$ of this suspension was plated on standardized Müller-Hinton agar for antimicrobial susceptibility testing (Becton Dickinson, USA). Etest ${ }^{\circledR}$ reagent strips were placed on the plates followed by aerobic incubation for $24 \mathrm{~h}$ at $34^{\circ} \mathrm{C}$.

Table 3: Antibiotics used for antimicrobial susceptibility tests, see also (14).

\begin{tabular}{|c|c|c|c|c|}
\hline $\begin{array}{l}\text { Antibiotic } \\
\text { substance }\end{array}$ & Type & $\begin{array}{l}\text { Mechanism of } \\
\text { action/target }\end{array}$ & Target group & $\begin{array}{l}\text { Concentrations } \\
\text { applied }(\mu \mathrm{g} / \mathrm{ml})\end{array}$ \\
\hline $\begin{array}{l}\text { Amoxicillin/ } \\
\text { clavulanic acid }\end{array}$ & $\begin{array}{l}\beta \text { - Lactam antibiotic } \\
\text { (penicillin) and } \beta \text { - } \\
\text { lactamase inhibitor }\end{array}$ & $\begin{array}{l}\text { Inhibits cell wall synthesis; } \\
\text { bactericidal }\end{array}$ & Gram + /- & $0.016-256$ \\
\hline Ampicillin & $\begin{array}{l}\beta \text { - Lactam antibiotic } \\
\text { (penicillin) }\end{array}$ & $\begin{array}{l}\text { Inhibits cell wall synthesis; } \\
\text { bactericidal }\end{array}$ & Gram + /- & $0.016-256$ \\
\hline Cefotaxime & $\begin{array}{l}\beta \text { - Lactam antibiotic } \\
\text { (cephalosporin) }\end{array}$ & $\begin{array}{l}\text { Inhibits cell wall synthesis; } \\
\text { bactericidal }\end{array}$ & Gram + /- & $0.016-32$ \\
\hline Ceftriaxone & $\begin{array}{l}\beta \text { - Lactam antibiotic; } \\
\text { (cephalosporin) }\end{array}$ & $\begin{array}{l}\text { Inhibits cell wall synthesis; } \\
\text { bactericidal }\end{array}$ & Gram + /- & $0.016-256$ \\
\hline Ciprofloxacin & Fluoroquinolone & $\begin{array}{l}\text { Inhibits bacterial DNA } \\
\text { gyrase; bactericidal }\end{array}$ & Gram + /- & $0.002-32$ \\
\hline Clarithromycin & Macrolide & $\begin{array}{l}\text { Inhibits protein synthesis; } \\
\text { bacteriostatic }\end{array}$ & Gram + /- & $0.016-256$ \\
\hline Clindamycin & Lincosamide & $\begin{array}{l}\text { Inhibits protein synthesis; } \\
\text { bacteriostatic }\end{array}$ & Gram + /- & $0.016-256$ \\
\hline Colistin & $\begin{array}{l}\text { Polypeptide antibiotic; } \\
\text { polymyxin }\end{array}$ & $\begin{array}{l}\text { Attacks cell membrane; } \\
\text { bactericidal }\end{array}$ & Gram - & $0.016-256$ \\
\hline Doxycycline & $\begin{array}{l}\text { Polyketide antibiotic; } \\
\text { (tetracycline) }\end{array}$ & $\begin{array}{l}\text { Inhibits protein synthesis; } \\
\text { bacteriostatic }\end{array}$ & Gram + /- & $0.016-256$ \\
\hline Gentamicin & Aminoglycoside & $\begin{array}{l}\text { Inhibits protein synthesis; } \\
\text { bactericidal }\end{array}$ & Gram + /- & $0.016-256$ \\
\hline Levofloxacin & Fluoroquinolone & $\begin{array}{l}\text { Inhibits bacterial DNA } \\
\text { gyrase; bactericidal }\end{array}$ & Gram + /- & $0.002-32$ \\
\hline Linezolid & Oxazolidinone & $\begin{array}{l}\text { Inhibits protein synthesis; } \\
\text { bacteriostatic }\end{array}$ & Gram + & $0.016-256$ \\
\hline Meropenem & $\begin{array}{l}\beta \text { - Lactam antibiotic } \\
\text { (carbapenem) }\end{array}$ & $\begin{array}{l}\text { Inhibits cell wall synthesis; } \\
\text { bactericidal }\end{array}$ & Gram + /- & $0.002-32$ \\
\hline Moxifloxacin & Fluoroquinolone & $\begin{array}{l}\text { Inhibits bacterial DNA } \\
\text { gyrase; bactericidal }\end{array}$ & Gram + /- & $0.002-32$ \\
\hline Penicillin G & $\begin{array}{l}\beta \text { - Lactam antibiotic } \\
\text { (penicillin) }\end{array}$ & $\begin{array}{l}\text { Inhibits cell wall synthesis; } \\
\text { bactericidal }\end{array}$ & Gram+ & $0.016-256$ \\
\hline $\begin{array}{l}\text { Trimethoprim/sul } \\
\text { famethoxazole }\end{array}$ & $\begin{array}{l}\text { Dihydrofolate } \\
\text { reductase inhibtor and } \\
\text { sulfonamide }\end{array}$ & $\begin{array}{l}\text { Inhibits tetrahydrofolate } \\
\text { synthesis; bactericidal }\end{array}$ & Gram + /- & $0.002-32$ \\
\hline Vancomycin & Glycopeptide antibiotic & $\begin{array}{l}\text { Inhibits cell wall synthesis; } \\
\text { bactericidal }\end{array}$ & Gram+ & $0.016-256$ \\
\hline
\end{tabular}

262 Co-incubation experiments and electron microscopy. To test if some of our isolates interact with, 
material. Pieces of NOMEX ${ }^{\circledR}$ fabric were provided by the Biotechnology Space Support Center (BIOTESC) of the Lucerne University and plates of the aluminum copper magnesium alloy EN AW 2219 which is also used on the ISS, were provided by Thales Alenia Space (TAS), Italy. NOMEX ${ }^{\circledR}$ is a flexible, flameproof fabric used for most storage bags aboard the ISS. The NOMEX ${ }^{\circledR}$ fabric was cut into pieces of $20 \mathrm{~mm} \times 30 \mathrm{~mm}$ and autoclaved before incubation. The aluminum alloy EN AW 2219 was cut into small plates of $20 \mathrm{~mm} \times 30 \mathrm{~mm} \times 3 \mathrm{~mm}$ by Josef Baumann in Falkenberg, Germany, and then evenly polished with a grit size of P240 and partly eloxated by Heuberger Eloxal, Austria. The autoclaved metal platelets, non-eloxated and eloxated, and NOMEX ${ }^{\circledR}$ fabric pieces were then incubated together with bacteria isolated from the ISS: Cupriavidus metallidurans pH5_R2_1_IIA (aerobic), Bacillus licheniformis R2A_5R_0.5 (aerobic), and Cutibacterium avidum R7A_A1_IIIA (anaerobic). Incubations were done in triplicates over a period of 3 months in liquid R2A medium in Hungate tubes at $\mathrm{pH} 7$ and $32^{\circ} \mathrm{C}$. Every 2 weeks, $50 \%$ of the medium was exchanged to ensure survival and further growth of the bacteria. After incubation, metal plates and NOMEX ${ }^{\circledR}$ fabric pieces were investigated via scanning electron microscopy. Metal plates and NOMEX ${ }^{\circledR}$ fabric pieces from the co-incubation experiment with selected bacteria were aseptically removed from their respective Hungate tube, carefully rinsed with 1xPBS buffer and then fixated overnight in a $100 \mathrm{mM}$ sodium cacodylate buffer containing $2.5 \%(\mathrm{v} / \mathrm{v})$ glutaraldehyde at $4^{\circ} \mathrm{C}$. Scanning electron microscopy of the samples was performed at the Biocenter of the Ludwig-Maximilians-University Munich using a Zeiss Auriga cross beam unit (Zeiss, Oberkochen, Germany).

Amplicon sequencing: Library preparation and sequencing were carried out at the Core Facility Molecular Biology at the Center for Medical Research at the Medical University Graz, Austria. In brief, DNA concentrations were normalized using a SequalPrep ${ }^{T M}$ normalization plate (Invitrogen), and each sample was indexed with a unique barcode sequence ( 8 cycles index PCR). After pooling of the indexed samples, a gel cut was carried out to purify the products of the index PCR. Sequencing was performed using the Illumina MiSeq device and MS-102-3003 MiSeq $^{\circledR}$ Reagent Kit v3-600cycles ( $2 \times 251$ cycles).

Sequence data processing and analysis: Demultiplexed, paired reads were processed in $\mathrm{R}$ (version 3.2.2) using the R package DADA2 as described in (51). In brief, sequences were quality checked, filtered, and trimmed to a consistent length of $\sim 270$ bp (universal primer set) and $\sim 140$ bp (archaeal primer set). The trimming and filtering were performed on paired end reads with a maximum of two expected errors per read ( $\operatorname{maxEE}=2$ ). Passed sequences were de-replicated and subjected to the DADA2 algorithm to identify indel-mutations and substitutions. The DADA2 output table is not based on a clustering step and thus no operational taxonomic units (OTUs) were generated. Each row in the 
taxonomic classification (ribosomal sequence variants; RSVs) (51). In addition, the merging step occurs after denoising, which increases accuracy. After merging paired end reads and chimera filtering, taxonomy was assigned with the RDP classifier and the SILVA v.123 trainset (52). The visualization was carried out using the online software suite "Calypso" (53). For bar plots data was normalized by total sum normalization (TSS) and for PCOA and Shannon index by TSS combined with square root transformation. Tax4fun was performed based on the Silva-classified OTU table, as described (54).

\section{RSV Network}

G-test for independence and edge weights were calculated on the RSV table using the make_otu_network.py script in QIIME 1.9.1 (55). The network table with calculated statistics was then imported into Cytoscape 3.7.1 (56) and visualized as a bipartite network of sample (hexagons) and RSV nodes (circles) connected by edges. For clustering, a stochastic spring-embedded algorithm based on the calculated edge weights was used. Size, transparency and labels were correlated with RSV abundances, border line intensity refers to RSV persistence over multiple sampling sessions and edge transparency was correlated to calculated edge weights.

Shotgun metagenomics. Shotgun libraries for Illumina MiSeq sequencing were prepared with the NEBNext ${ }^{\circledR}$ Ultra II DNA Library Prep Kit for Illumina ${ }^{\circledR}$ in combination with the Index Primer Set 1 (NEB, Frankfurt, Germany) according to manufacturer's instructions and as described in (57). Briefly, $500 \mathrm{ng}$ of dsDNA were randomly fragmented by ultrasonication in a microTUBE on a M220 Focusedultrasonicator $^{\mathrm{TM}}$ (Covaris, USA) in a total volume of $130 \mu \mathrm{l}$ 1xTE for 80 seconds with 200 cycles per burst (140 peak incident power, 10\% duty factor). After shearing, $200 \mathrm{ng}$ of sheared DNA were used for the end repair and adapter ligation reactions in the NEBNext ${ }^{\circledR}$ Ultra II DNA Library Prep Kit for Illumina ${ }^{\circledR}$ according to manufacturer's instructions. Size selection and purification were performed according to the instructions for 300 to $400 \mathrm{bp}$ insert size. Subsequent PCR amplification was performed with 4 cycles and libraries were eluted after successful amplification and purification in 33 $\mu \mathrm{l}$ 1xTE buffer pH 8.0. For quality control libraries were analyzed with a DNA High Sensitivity Kit on a 2100 Bioanalyzer system (Agilent Technologies, USA) and again quantified on a Quantus ${ }^{\mathrm{TM}}$ Fluorometer (Promega, Germany). An equimolar pool was sequenced on an Illumina MiSeq desktop sequencer (Illumina, CA, USA). Libraries were diluted to $8 \mathrm{pM}$ and run with 5\% PhiX and v3 600 cycles chemistry according to manufacturer's instructions. Raw fastq data files were uploaded to the metagenomics analysis server (MG-RAST) (58) and processed with default parameters. Annotations of taxonomy (RefSeq) and functions (Subsystems) were then imported to QIIME 2 (2018.11) (59) or visualizations of the datasets. 
332 Controls. Cultivation, extraction, PCR, and sequencing controls were processed and analysed in 333 parallel to biological samples. An unused wipe not taken out of its bag on the ISS was extracted for 334 every sampling session, cut into pieces, placed on the different media and DNA was also extracted 335 from the solutions obtained with the negative controls. All cultivation controls were negative (no 336 growth of colonies). Wipe solutions of the negative controls used for DNA extraction, PCR, and sequencing revealed a low number of ribosomal sequence variants. These RSVs were removed from according datasets, if present in the samples.

Data availability. Data are available on request. 
In-flight sampling on board the ISS was performed during increment 51 and 52 (April to June 2017) under the ESA operation named EXTREMOPHILES. All samples ( $n=24$, plus controls) were taken by US astronaut Jack D. Fischer. Three sampling sessions were performed, with session A on 01.05.2017, session $B$ on 12.07.2017 and session C on 29.06.2017. With a span of 72 days between session $A$ and B they were conceptualized for time-course sampling (same sampling locations) to get an idea on the microbiome fluctuation. For comparative purposes, an ISS-relevant cleanroom and a therein housed cargo-spacecraft were sampled, namely cleanroom S5C at the Centre Spatial Guayanais near Kourou in French Guiana with ATV spacecraft.

ISS microbiome is dominated by human-associated microorganisms and contains also archaeal signatures.

The microbial community composition was assessed by amplicon sequence analysis of wipe samples obtained from sessions $A-C$ and the Kourou cleanroom. After complete removal of RSVs from negative controls, more than 3.500 RSVs remained from all ISS and cleanroom samples for the analysis. Both, archaeal and bacterial signatures were retrieved from ISS samples with the universal approach (Fig 1). The signatures belonged to 377 genera, with Streptococcus, Corynebacterium, Lactobacillus, Acinetobacter, Staphylococcus predominating (Fig. 1). Firmicutes, Proteobacteria, Actinobacteria and Bacteroidetes were found to be the predominant bacterial phyla (all samples), whereas archaeal signatures (Woesearchaeota, Thaumarchaeota, Euryarchaeota) were frequently detected in air (Cupola air 14.1\% and Columbus air session B 3.6\% of all sequences) and on various surfaces (Fig. 1). By the archaea-targeting approach, mostly gut associated Methanobrevibacter sequences (surface Cupola [sample C2], waste and hygiene compartment [C5]), Woesearchaeota (ATU [A8, B8]; hand grips Columbus [B4]), and unclassified Archaea (ATU [A8], dining table [C7]) were detected. To a lesser extent we also found signatures from Halobacteria (SSC Laptop Columbus [A2]) and Thaumarchaeota (dining table [C7]). 

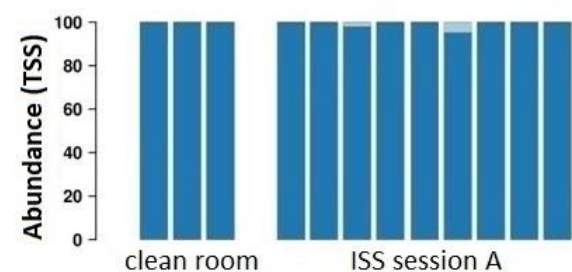

ISS session A
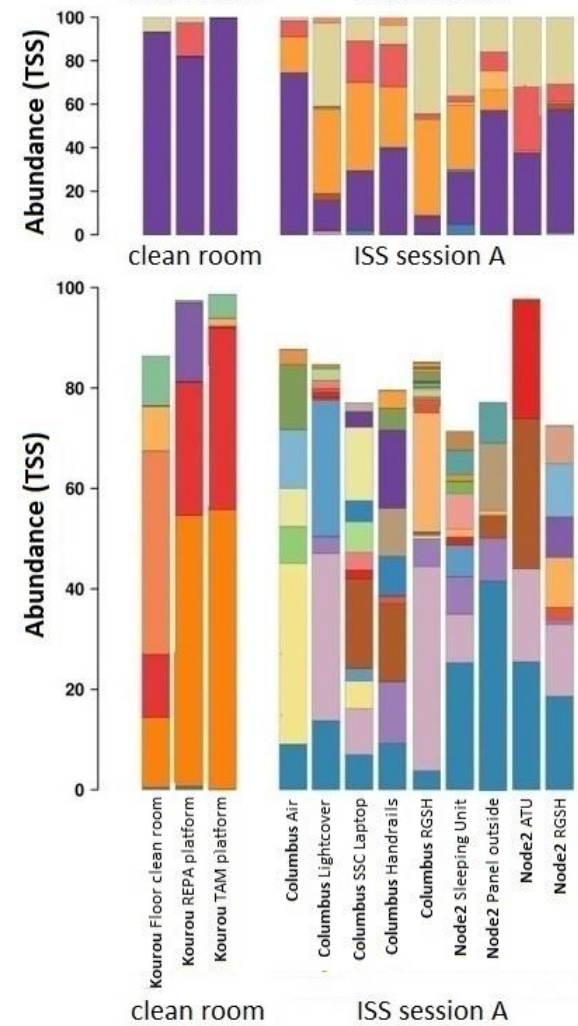

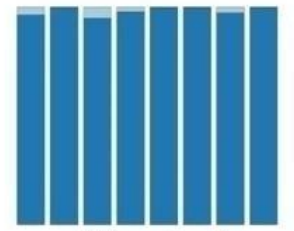

ISS session B

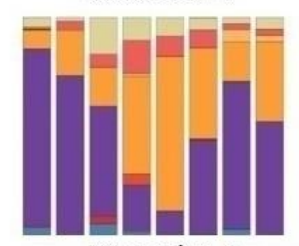

ISS session B

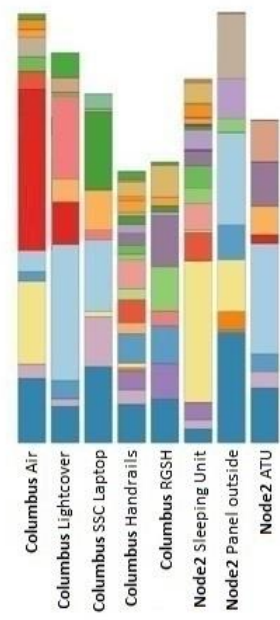

ISS session B

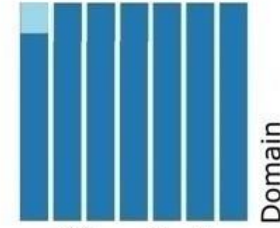

ISS session C

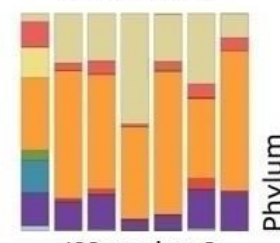

ISS session C

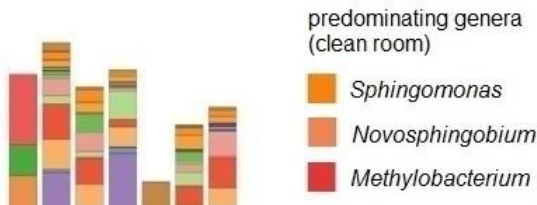

predominating genera (ISS)

Streptococcus

Corynebacterium

Lactobacillus

Acinetobacter

Staphylococcus

unknown

Fig. 1: Microbiome composition in cleanrooms (left column) and the ISS sampled during session A, B and C.

Microbiome dynamics (comparison of session $A$ and $B$ ). To retrieve insights into the microbiome dynamics over time, session A and B were conceptualized with a time-lapse of 72 days between the two samplings. During the complete sampling timeframe, no crew exchange took place, but two cargo deliveries docked (SpaceX and Soyuz). Notably, the microbial diversity (RSVs) were found to be increased significantly in samples of session B ( $p=0.034$, ANOVA; Inverse Simpson's, rarefied to a depth of 649 RSVs; Fig. 2a), however, the evenness of samples did not change significantly ( $p=0.68$, ANOVA). Anosim analysis indicated a significantly different composition of the samples taken in session $A$ and $B(p=0.001)$. LEfSe analysis (targeting the 300 most abundant genera) identified a substantial increase of signatures belonging to typically gastrointestinal tract-associated genera Escherichia/Shigella ( $p=0.017$, ANOVA), Lachnoclostridium, Ruminococcus_2 $(p=0.046)$ and 

unclassified Corynebacteriaceae $(p=0.02)$ were significantly reduced. of Ralstonia in samples of session B. Pie charts were created from single locations within the ISS to visualize the changes in microbiome composition (Fig. 2c). It shall be noted, that signatures of unclassified Woesearchaeota (DHVEG6) were found amongst the 40 most abundant microbial genera (additional details below).
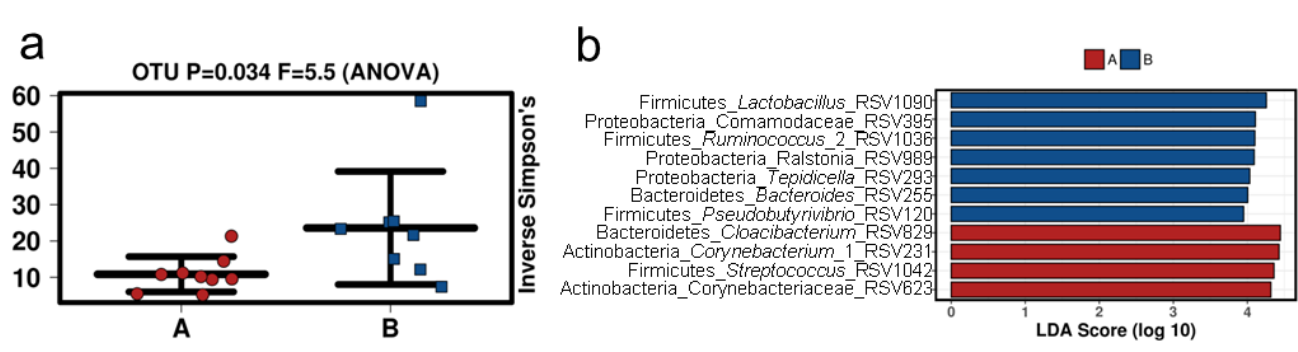

C

Columbus Air - $\mathrm{A}$ Columbus Air -B
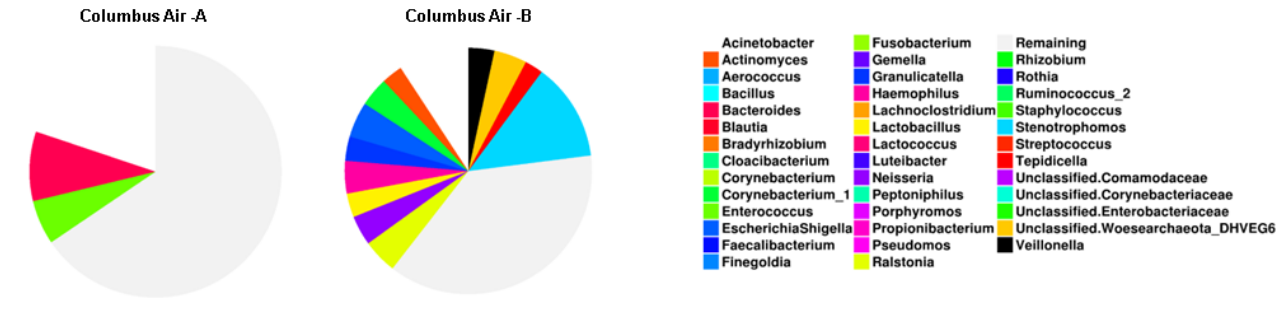

Columbus Handrail-A
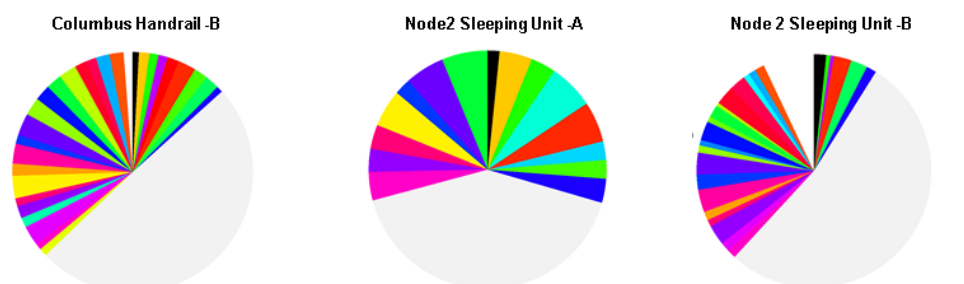

Fig. 2: Microbiome diversity and dynamics in samples from sampling session $A$ and $B$ (same locations were sampled, universal approach). a) Inverse Simpson's index, indicating a significantly different microbiome diversity in samples from session A and B. b) LEfSe analysis (300 most abundant taxa), comparing session A and B. c) Pie charts created including the top $\mathbf{4 0}$ most abundant microbial taxa for selected samples. "Columbus Air - $A$ " refers to sample taken from Columbus module: air, in session $\mathrm{A}$.

\section{The ISS harbors a core microbiome of more than $\mathbf{5 0}$ microbial genera.}

Core microbiome analyses, looking at the 100 most abundant RSVs, identified 34 taxa shared amongst all sampling time points (A-C, minimal relative abundance: 10\%), whereas 55 taxa were shared on genus level. The most abundant, shared RSVs belonged to the microbial genera Haemophilus, Gemella, Streptococcus, Corynebacterium, Staphylococcus, Lactococcus, Neisseria and 


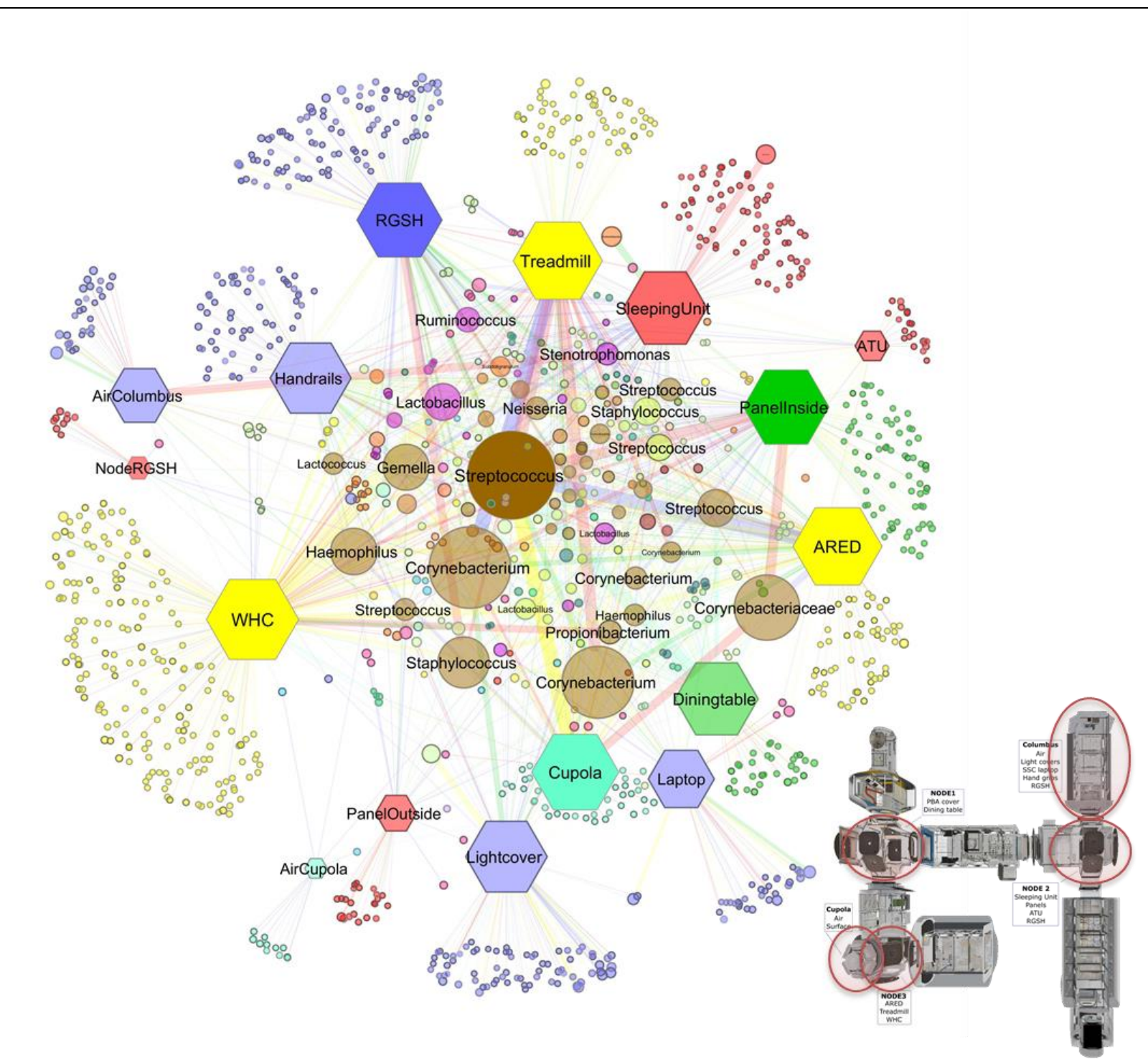

Fig. 3: Network analysis and model of the ISS with sampling sites (red circles). Samples are shown as hexagons, RSVs are shown as circles. Color of sample nodes refer to respective ISS modules. RSV nodes show mixtures of these colors if they were shared by several locations and modules of the ISS. The borders of the circles are darker, when found in several sessions. The size, transparency and the size of the node descriptions corresponds to their abundance. The thickness and transparency of the edges (lines) follows the calculated e-weights. Layout: Spring Embedded, with e-weights. Colour code: Yellow (NODE3), red (NODE2), blue (Columbus), aqua (Cupola), green (NODE1). Abbreviations: WHC (Waste and hygiene compartment), ARED (Advanced Resistive Exercise Device), ATU (Audio terminal unit), RGSH (Return Grid Sensor Housing). ISS model source: NASA (https://nasa3d.arc.nasa.gov/detail/iss-internal, accessed on Jan 2019)

414 The network analysis showed a higher abundance for RSVs which belong to the core ISS microbiome (e.g. Streptococcus, Corynebacterium, Staphyloccocus, Haemophilus, Gemella or Propionibacterium).

Notably, most location-specific RSVs were observed for WHC (waste and hygiene compartment) and RGSH (return grid sensor housing). This was expected for the WHC area (as hygienic activities shed 418 (internal) human microorganisms into the environment), but surprising for the RGSH. The RGSH is 
the air inlet part of the air recycling system, expected to accumulate biological burden from the environment, but not to host indigenous microbiology.

Locations with regular crew activity (e.g. treadmill, sleeping unit, handrails) showed higher proportions of RSVs assigned to the human-associated genera Stenotrophomonas, Ruminococcus and Lactobacillus. When clustering the samples according to their origin, the network also indicated that locations exposed to high human traffic from different modules are more similar to each other than samples of high and low human traffic which were taken within the same ISS module.

\section{Location shapes microbiome composition}

In a next analyses step, we were interested in external parameters influencing the ISS microbiome. Redundancy analysis indicated a significant effect of the time of sampling (sessions; $p=0.010$ ), and indicated a potential effect of the location within the ISS (module; $p=0.054$ ) on microbiome composition. We further categorized the different samples into: air, personal area (sleeping unit), shared areas which are highly frequented (e.g. communication items, handrails), and shared areas which are less often touched (e.g. lightcover, RGSH, etc.). An NMDS plot performed on RSV level indicated a different composition of the microbiome according to these categories, whereas shared surfaces showed an overlap no matter whether they were frequently or less frequently touched. However, this grouping was not confirmed by statistical analyses $(p=0.364$, Anosim based on BrayCurtis distance metric). The highest diversity of microbial signatures was detected in personal areas of the ISS, without being significantly different.
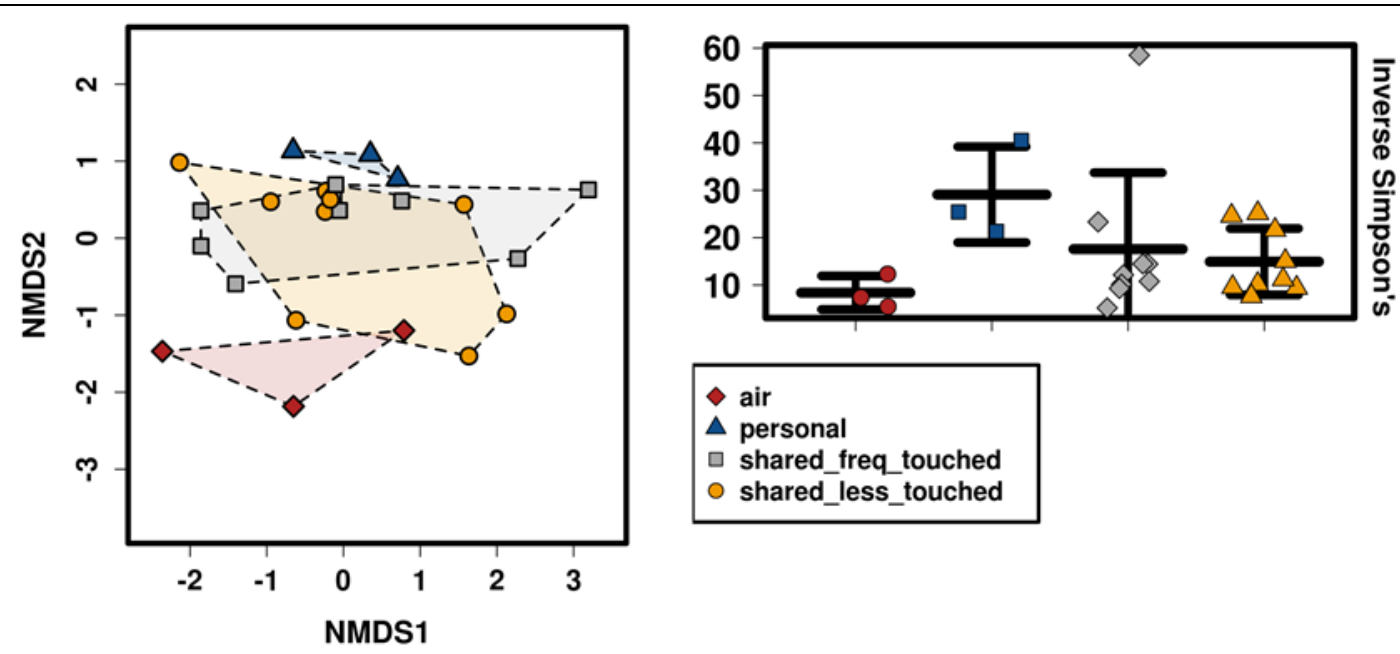

Fig. 4: Microbiome composition according to sample categories. Left: NMDS plot (stress 0.129 ) indicating a separate grouping of personal microbiomes; right: the highest diversity of microbial signatures was observed in microbiome samples from personal areas. The lowest diversity was detected in air samples ( $p=0.19$, Anova; sample depth rarefied to 649 reads). 
ANOVA plot analysis indicated e.g. the increased presence of human-associated Streptococcus RSVs in samples from dining table and workout area, Neisseria species (human mucosa-associated microorganisms) were particularly detected in the sleeping unit and work out area. Lactococcus signatures were particularly found in samples from the dining table (potentially food-associated), the sleeping unit and the workout area, whereas RSVs from Actinomyces, Enterococcus, Lautropia and Brevibacterium were significantly enriched on handrails, in air, on the dining table, and in the work out area, respectively (all p-values $<0.05$, see Fig. 5). The waste and hygiene area showed significantly increased abundances of RSVs belonging to Lactobacillus, Propionibacterium, Collinsella, Subdoligranulum, Romboutsia and Anaerostipes (Fig. 5). All in all, the samples retrieved from the ISS were largely reflecting the human microbiome, as other sources of the microbial signatures could not be identified (besides potentially food for Lactococcus).

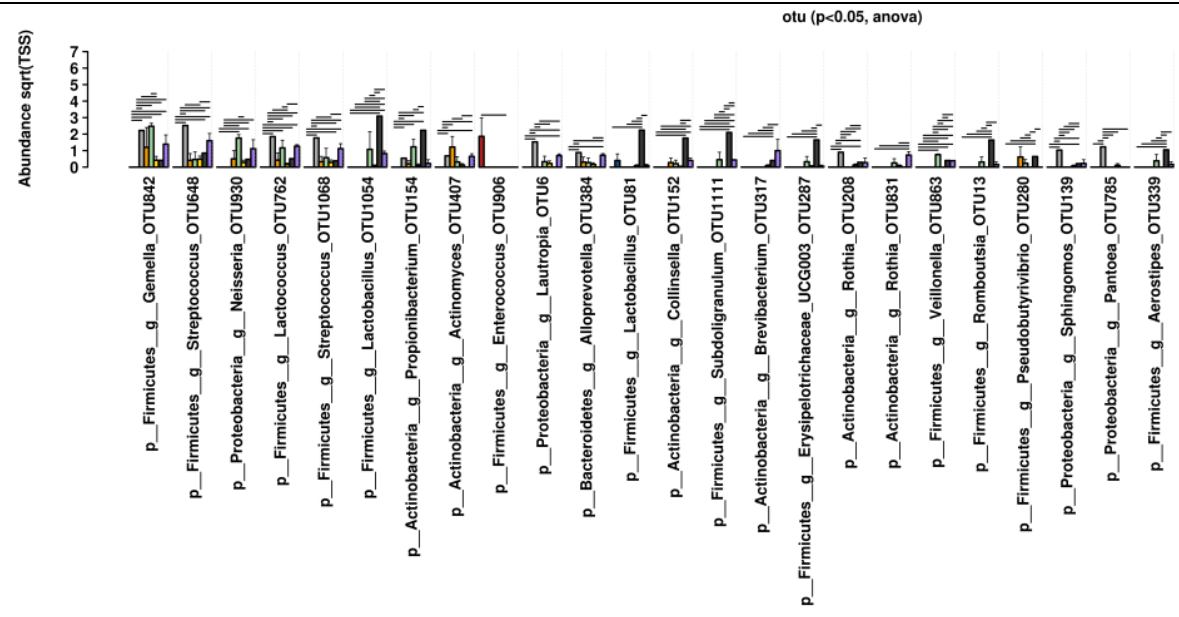

Fig. 5: ANOVA plot analysis of different types of locations within the ISS and specifically associated RSVs.

According to a hierarchical cluster analysis based on Pearson's correlation across session A and B (Fig. 6), a positive correlation of certain microbial phyla with sampled locations was found, being in agreement with findings from the cultivation assays (e.g. Deinococcus sp. was isolated from Node2_Panel_Outside). Columbus handrails were found to be correlated with e.g. Saccharibacteria and Bacteroidetes. A particular pattern was detected for the archaeal signatures retrieved, which were found to be indicative for the sleeping unit (Thaumarchaeota), the handrails (Euryarchaeota) and the samples from the Columbus_SSC_Laptop (Woesearchaeota). 

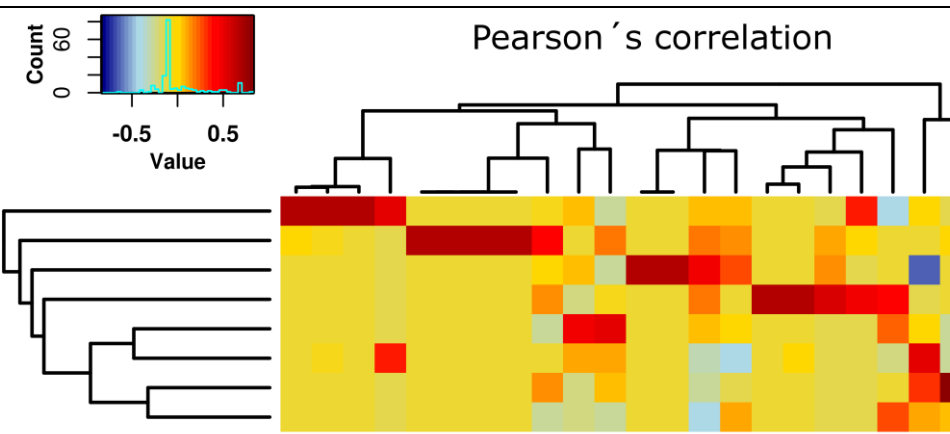

Columbus_Lightcover

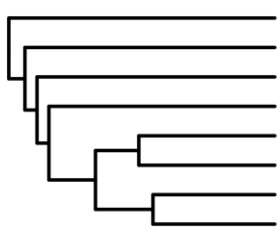

Node2_Sleeping_Unit

Columbus RGSH

Columbus_Handrails

Columbus_SSC_Laptop

Columbus Air

Node2 Pañel outside

Node2_ATU

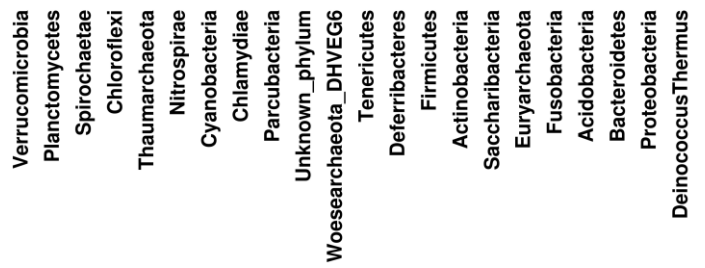

Fig. 6: Hierarchical cluster analysis (Pearson's correlation; universal microbiome data set). Certain microbial phyla (in particular archaeal phyla) were found to correlate with specific sampling sites.

\section{Microbial functions inferred from 16S rRNA gene information (Tax4fun) and metagenomic analyses}

As metagenomics could only be performed on pooled subset of samples (due to low biomass restrictions and sampling set-up, see below), Tax4fun analysis was initially used to predict potential microbial metabolic capabilities, their location-specificity and potential shifts over time.

LEfSe analysis (Fig. 7c) revealed a location specific predicted capacity of the microbial community, with e.g. increased predicted functions in KEGG pathway "Base_excision_repair" in samples from the cupola module, a probably indicator for elevated radiation levels.

On gene level, different functions were predicted, indicative of a respective module. Node 3 (ARED, treadmill and WHC) revealed predicted signatures of cobalt/nickel and antibiotic transport system ATP binding proteins. Notably, a specific increase of a cobalt/zinc/cadmium resistance protein was predicted for Node 2 (sleeping area and panel samples), an iron complex transport system substrate binding protein for Node 1 (e.g. dining area), and an antibiotic transport system permease protein for the cupola area. This indicated a potential microbial competition and a relevance of transition metal components (e.g. from ISS materials) for the microbial community in these environments. 


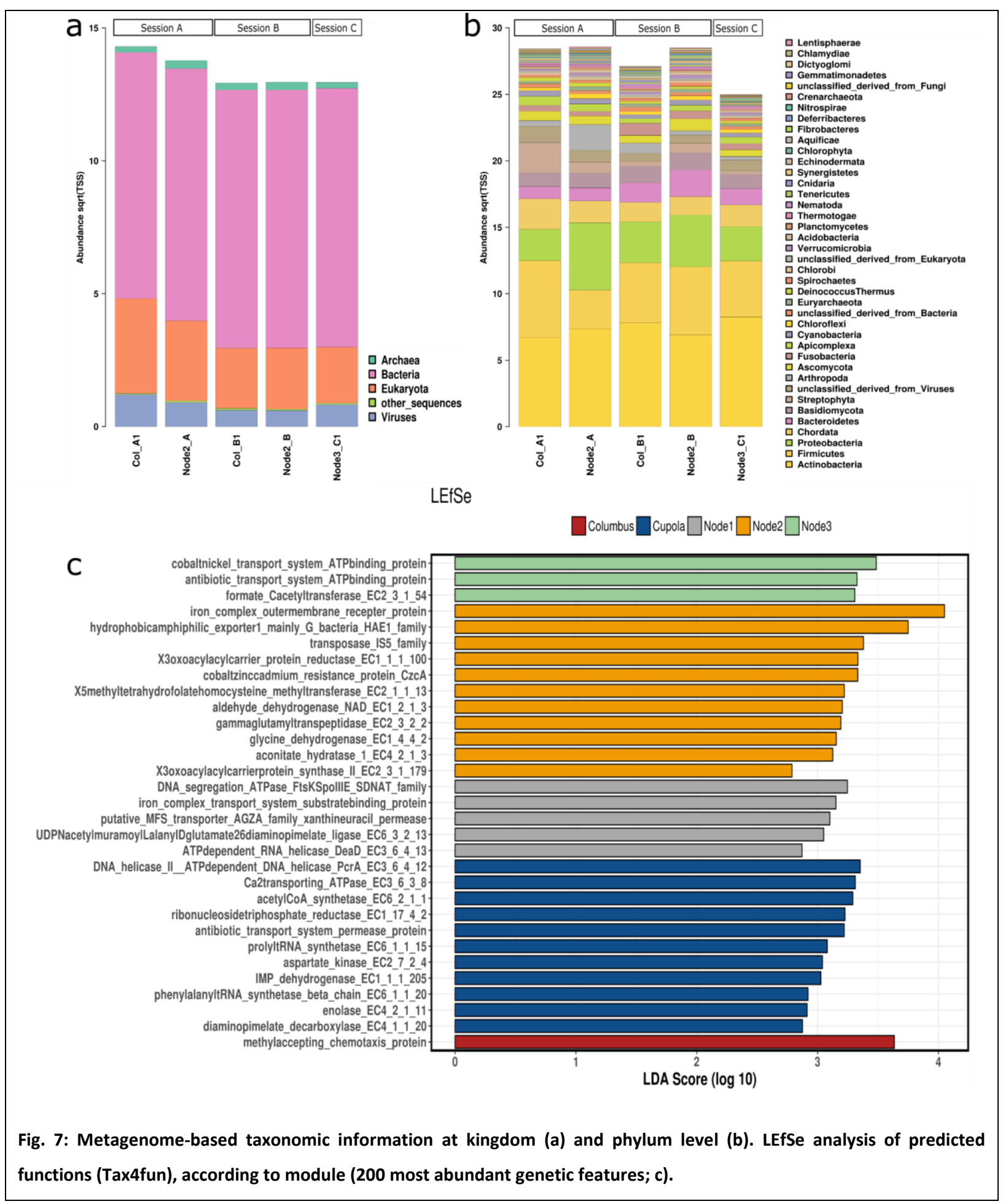


For metagenomics, samples were pooled as follows: All samples from Columbus module session A (COLA), all samples from Columbus module session B (COLB), all samples Node 1 session C (N1C), all samples Node 2 session A (N2A), all samples Node 2 session B (N2B), all Node 3 session C (N3C).

The taxonomic composition as retrieved from metagenomics was found to be somewhat different to the amplicon-based analysis. In particular the predominance of Propionibacterium reads was striking, as its signatures were not well reflected in the amplicon approach. However, Staphylococcus, Corynebacterium, Streptococcus could be confirmed as omnipresent on all sampled surfaces of the ISS. However, fungal (e.g. Malassezia), viral (e.g. Microvirus) and archaeal sequences (e.g. Methanobrevibacter) also belong to the core taxa of the ISS (Fig. 7a,b). Core microbial taxa and functions showed a stable distribution over different fractions of samples. Hence, $57 \%$ of all taxa and $34 \%$ of all functions were shared in all samples (even higher proportions were visible when samples were grouped per module $68 \%$ and $51 \%$ or per sampling session $73 \%$ and $58 \%$ respectively). the Columbus module also experienced the biggest shift of its microbiome along PCoA Axis 1 (taxa: $\sim 60 \%$, functions $\sim 40 \%$ ) between the first and last sampling session.

Regarding functions, genes assigned to arginine biosynthesis (amino acid metabolism), degradation of L-Ornithine (amino acid metabolism), copper-translocating P-type ATPases (virulence, disease and defense), and the phage major capsid protein (phages, prophages, transposable elements) were ubiquitously distributed, whereas functions assigned to dormancy and sporulation, photosynthesis, motility and chemotaxis as well as aromatic compounds metabolism showed location-dependent variations. Functions involved in iron acquisition and metabolism (ferrous iron transport protein B: $0.2 \%$ in functional core), potassium metabolism, nickel $A B C$ transporters and others were highly abundant, indicating a potential surface-interaction with ISS materials.

\section{ISS cultivable microbial community reveals extremo-tolerant traits}

In the course of this study, hundreds of colonies/cultures retrieved on/in various media were processed, resulting in 76 unique bacterial isolates. Along with the bacteria, also fungi were isolated, but are not further analyzed herein. These included Aspergillus species (A. sydowii, A. unguis),

516 Chaetomium globosum, Penicillium species (P. aurantiogriseum, P. brevicompactum, P. chrysogenum, 517 P. crustosum, P. expansum), Rhizopus stolonifera and Rhodotorula mucilaginosa. All fungal isolates 
obtained in this study were assigned to biosafety risk group S1. P. brevicompactum, P. chrysogenum, can also act as opportunistic pathogen. Archaea could not be grown from any sampling site. growth/enrichment characteristics, which is shown in Fig. 8.

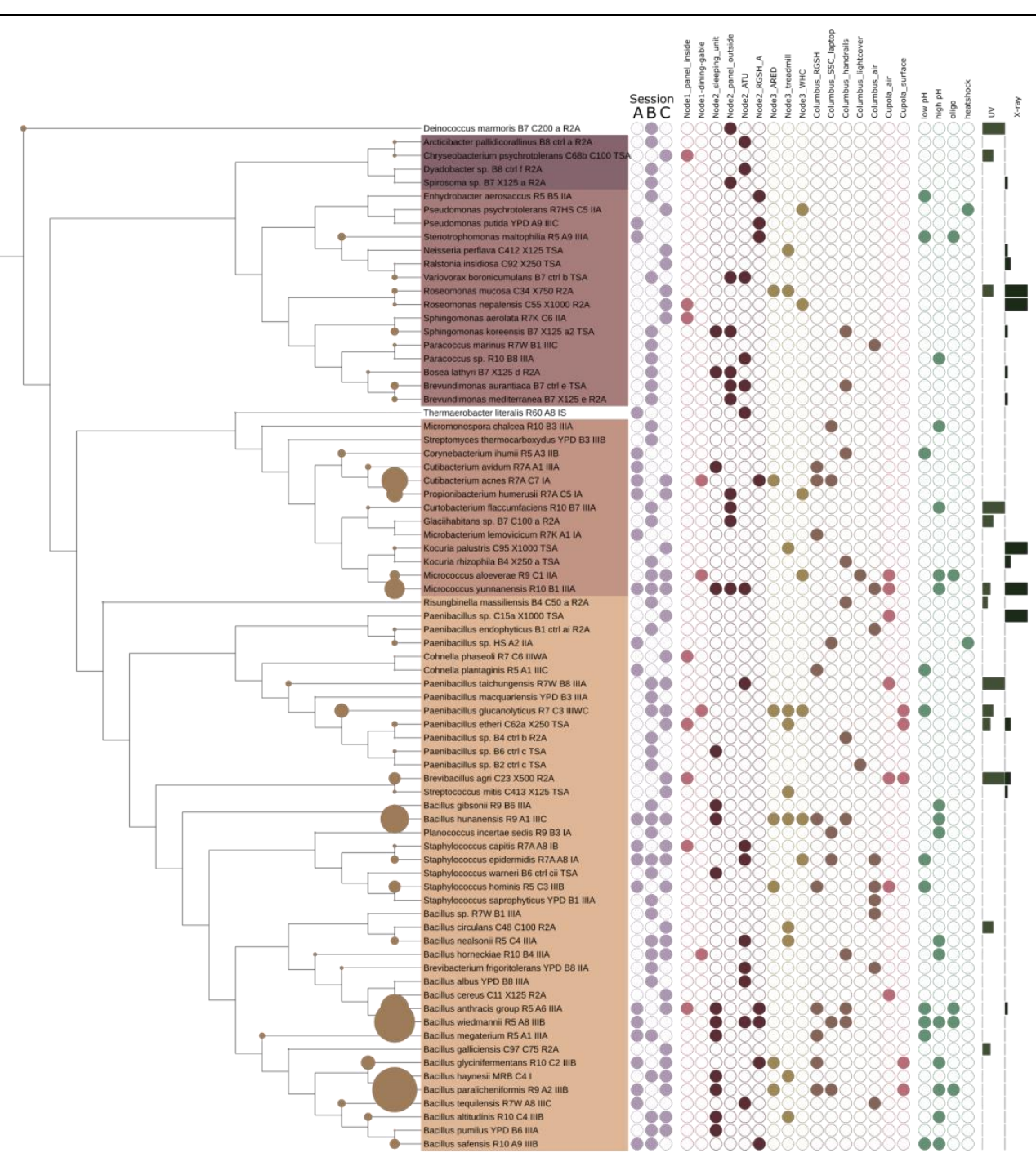

Fig. 8: Tree of all isolates retrieved from sessions A-C. Circles on branches reflect the number of isolates obtained from the respective species. First dataset reflects the session number $(A-C)$, the locations where the isolates were retrieved from (Node 1, 2, 3, Columbus and Cupola in different colors), the enrichment conditions (low, high pH, oligotrophic conditions and heat-shock resistant) and the radiation dose (UVC, X-Ray; bars on the right) which were used to pre-treat the samples.

It shall be noted, that a number of isolates was obtained under stringent cultivation or pre-treatment conditions. This included i) UV- and X-ray resistant microorganisms, such as Deinococcus marmoris, Curtobacterium flaccumfaciens, Brevibacillus agri $\left(\mathrm{UV}_{254 \mathrm{~nm}}: 200 \mathrm{~J} / \mathrm{m}^{2}\right)$, Roseomonas species, Kocuria palustris, Micrococcus yunnanensis, Paenibacillus sp. (X-ray: $1000 \mathrm{~Gy}$ ), ii) microorganisms growing particularly at high or low $\mathrm{pH}$, or iii) heat-shock survivors (Pseudomonas psychrotolerans, 
Paenibacillus sp.) (Fig. 7). Isolates retrieved under non-mesophile conditions were, for example, Thermaerobacter literalis (a true thermophile isolated at $65^{\circ} \mathrm{C}$ from the ATU in Node2, does not grow below $50^{\circ} \mathrm{C}$ ), Sphingomonas aerolata and Microbacterium lemovicicum (exhibiting extraordinary cryotolerance, isolated only at $4^{\circ} \mathrm{C}$, maximal growth temperatures were $51^{\circ} \mathrm{C}$ and $32^{\circ} \mathrm{C}$, respectively).

\section{Physiological characteristics and resistance potential of ISS microbes do not differ from ground controls.}

In the next step, we analyzed physiological characteristics of ISS microbial isolates. In particular, we were interested whether they withstand physical and chemical stressors better than same or closely related microbial species from ground controls.

For these tests, we selected a subset of microbial isolates from the ISS, spanning 11 microbial genera of interest (listed in Fig. 9). This list included typical confined-indoor bacteria, like Bacillus, Micrococcus and Staphylococcus, but also microorganisms of special interest (associated to spacecraft assembly, extraordinary hardy, extremophile) were included (e.g. Microbacterium, Cupriavidus, or Ralstonia). For comparative reasons, we included also eight microbial isolates from ground controls (cleanrooms, Concordia station) or culture collections. Overall, the final list comprised 29 different microbial strains.

All these strains were tested with respect to heat-shock resistance in the stationary phase (Bacillus cultures contained spores), upper and lower temperature limit (growth), upper and lower pH limit (growth), and resistance towards a variety of antibiotics (Fig. 9).

Antimicrobial susceptibility testing for 17 clinically relevant antibiotics was performed. Antibiotic resistance/susceptibility was found to be in some cases strain-specific but mostly species/genusspecific, independent from their isolation source (ISS or ground control). In particular the tested Bradyrhizobium species showed a vast resistance against numerous antibiotics, as did one Roseomonas strain. The antibiotic resistance pattern was judged following the EUCAST guidelines for PK/PD (non-species related) or, for Staphylococcus isolates, Staphylococcus spp. breakpoints (14) were used. It has to be stressed that all tested isolates were non-pathogenic and that these results shall not be used for clinical risk assessment of any kind. The ISS strains were not found to be significantly more resistant (number of antibiotics or concentration) than their counterparts from ground controls.

Notably, the strains showed a growth temperature span of 18 to 52 degrees $\left(14-32^{\circ} \mathrm{C}\right.$, Roseomonas C63; Bacillus pumilus, $4-56^{\circ} \mathrm{C}$ ). The minimal and maximal growth temperatures, or the temperature 
bioRxiv preprint doi: https://doi.org/10.1101/533752; this version posted January 29, 2019. The copyright holder for this preprint (which was not certified by peer review) is the author/funder. All rights reserved. No reuse allowed without permission. control strains was observed (Mann-Whitney U Test).

span, were not significantly different in ISS isolates compared to control microorganisms (MannWhitney $\mathrm{U}$ Test). For growth at different $\mathrm{pH}$ values, the isolates revealed a $\mathrm{pH}$ span of 4 to $10 \mathrm{pH}$ values (Bradyrhizobium erythroplei LMG28425, pH3-7; Bacillus altitudinis R10_C4_IIIB, pH 2-12). As seen for the temperature, no significant difference in $\mathrm{pH}$ preference of ISS strains versus ground

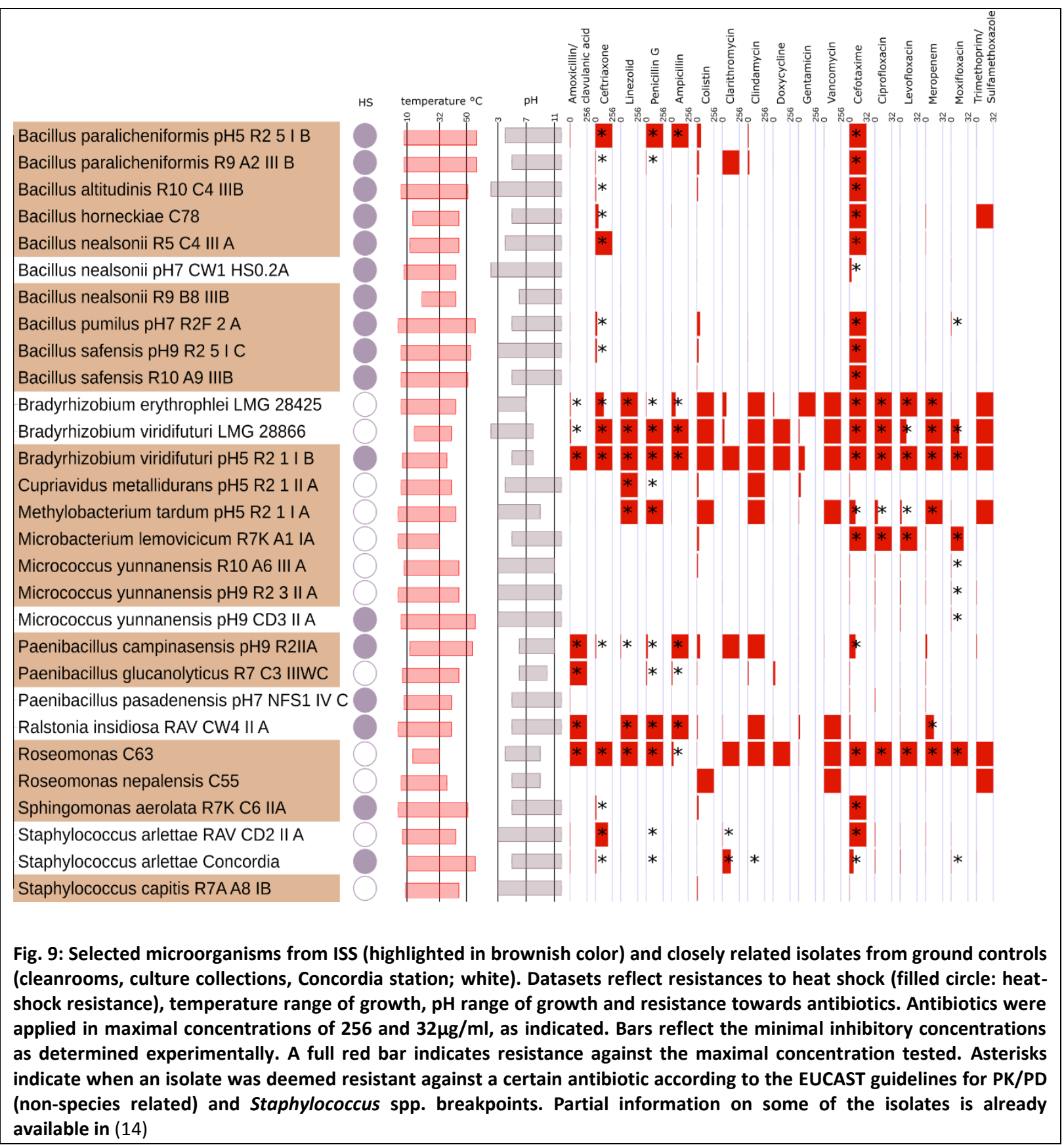



partial discrepancy of genomic and experimentally observed antibiotics resistance patterns

584

585

586

587

588

589

590

591

592

593

594

595

596

597

598

599

600

601

602

603

604

605

606

607

608

609

610

611

612

613

In order to understand the specific genomic characteristics of ISS microorganisms, we selected six different species for genome sequencing and reconstruction, namely: Bacillus pumilus strain pH7_R2F_2_A, Bacillus safensis strain pH9_R2_5_I_C. Bradyrhizobium viridifuturi strain pH5_R2_1_I_B, Cupriavidus metallidurans strain pH5_R2_1_II_A, Methylobacterium tardum strain pH5_R2_1_I_A and Paenibacillus campinasensis strain pH9_R2IIA (41) and compared the assemblies to publicly available genomes.

The genome of Bacillus pumilus strain pH7_R2F_2_A was retrieved $99.59 \%$ complete, with a \%GC of 41.6. The overall genome length was 3.7 Mbp. Bacillus pumilus SAFR-032 (3.7 Mbp, 41.3 \%GC; ENA study ID: PRJNA20391), whose genome was analysed for comparative reasons as well, possessed the same antibiotics resistance capacity. The ISS strain possessed all necessary genes for flagellum assembly and CAS-TypellIB (with cmr5_TypellIB missing); the latter was not found in Bacillus pumilus SAFR-032. Looking at the metabolic profiles, the ISS isolate of Bacillus pumilus (comparison to SAFR032 and ATCC 7061 (3.8 Mbp, 41.7 \%GC; ENA study ID: PRJNA29785) possessed the genomic capacity to perform choline and methionine degradation, but no other peculiarities were identified.

The genome of Bacillus safensis strain pH9_R2_5_I_C was found to be $99.59 \%$ complete, with a \%GC of 41.5. The overall genome length was $3.7 \mathrm{Mbp}$. It possessed all necessary genes for flagellum assembly and CAS-TypellIB, as did next neighbour Bacillus safensis FO-36b. Looking at the metabolic profiles, the ISS isolate of Bacillus safensis (comparison to CFA06 (3.7 Mbp, $41.5 \%$ GC; ENA Study ID: PRJNA246604) and FO-36b (3.7 Mbp, 41.6 \%GC; ENA Study ID: PRJNA270528) did not show certain peculiarities.

The genome of Bradyrhizobium viridifuturi strain pH5_R2_1_I_B was found to be $99.96 \%$ complete, with a \%GC of 64.3. The overall genome length was 7.9 Mbp. The genome carried several copies of the efflux pump membrane transporter BepG as well as other multidrug efflux transporters and $\beta$ lactamase genes, which largely explained the overall stable antibiotic resistance observed in our experiments. The observed resistances against linezolid and vancomycin could not be directly inferred from the genomic data. These features were also found in Bradyrhizobium viridifuturi SEMIA 690 (8.8 Mbp, 64.0 \%GC; ENA Study ID: PRJNA290320), the next phylogenetic neighbour. Overall, the genetic features of our ISS isolate were widely similar to known Bradyrhizobium species. The only differences found were the potential capability for homospermidine biosynthesis from putrescine and $(\mathrm{R})$-acetoin biosynthesis through (S)-2-acetolactate. 
The genome of Cupriavidus metallidurans strain pH5_R2_1_II_A was found to be $99.94 \%$ complete, with a GC content of $63.7 \%$. The overall genome length was $6.9 \mathrm{Mbp}$. This strain carries three bepE efflux pump membrane transporters, and also a multidrug efflux system protein (acrB). However, the bepE efflux pumps were not detected in the genome of its closest relative $C$. metallidurans $\mathrm{CH} 34$. The genome showed full potential for type IV pili and flagella formation and numerous secretion systems, but this was not a unique feature for the ISS strain. With respect to the metabolic profile, $C$. metallidurans strain pH5_R2_1_II_A showed a number of different features when being compared to the next relatives (C. basilensis OR16, ENA Study ID: PRJNA79047; C. metallidurans CH34, ENA Study ID: PRJNA250; C. necator N-1, ENA Study ID: PRJNA67893; C. taiwanensis LMG19424, ENA Study ID: PRJNA15733), which included the predicted capacity for 5,6-dimethylbenzimidazole biosynthesis and butanediol degradation/synthesis.

The genome of Methylobacterium tardum strain pH5_R2_1_I_A was found to be $100 \%$ complete with a GC content of $69.2 \%$, and a total genome length of $6.5 \mathrm{Mbp}$. Also M. tardum pH5_R2_1_I_A carried the efflux pump membrane transporter BepE and the genetic capacity for flagellum formation and several secretion systems. The strain showed a number of differential features when we compared the genomic potential with other members of the genus $(M$. extorquens, $M$. mesophilicum, $M$. nodulans, M. populi, M. radiotolerans; 5,6-dimethylbenzimidazole biosynthesis, base-degraded thiamine salvage, cytidylyl molybdenum cofactor biosynthesis, L-dopachrome biosynthesis); however, it shall be noted, that another genome of the species was not available at the time of analysis.

The genome of Paenibacillus campinasensis strain pH9_R2IIA could be retrieved with a $99.84 \%$ completeness. It showed a GC content of $52.26 \%$, and a genome length of $5.4 \mathrm{Mbp}$. The genome revealed a potential for lincosamide (Clindamycin), macrolide (clarithromycin), fluorquinolone (moxifloxacin, levofloxacin, ciprofloxacin), and glycopeptide (vancomycin) resistance which could all be verified by the antimicrobial susceptibility tests with the exception of the vancomycin resistance (no PK/PD breakpoint in the EUCAST table). Nevertheless, the observed MIC for vancomycin was 4 $\mu \mathrm{g} / \mathrm{ml}$, which was the highest observed MIC for vancomycin besides the seven isolates which were completely resistant (see Fig. 8). The genome did not show any $\beta$-lactam resistances but in spite of this, Paenibacillus campinasensis strain pH9_R2IIA was resistant against all $\beta$-lactam antibiotics with the exception of meropenem in the antimicrobial susceptibility tests. The strain showed the potential for flagella formation, and the presence of CAS type III. At the time of the analysis there was no other genome of this species publically available, but the metabolic potential was not found to be strikingly different from other genome-sequenced members of the Paenibacillus genus. 
The antibiotic resistance genes (ARG) detected in the sequenced genomes and the inferred antibiotic resistances are summarized in Fig. 10. These detected ARGs conformed for the most part with the results from the antimicrobial susceptibility tests; however, there were also some discrepancies. For example, both Bacillus strains had genes for the transcription-repair coupling factor $\mathrm{mfd}$ and the efflux transporter blt which should provide resistance against multiple fluorquinolones, but Bacillus pumilus strain pH7_R2F_2_A was only resistant against moxifloxacin and not against ciprofloxacin or levofloxain, while Bacillus safensis strain pH9_R2_5_I_C was sensitive against all tested fluorquinolones. C. metallidurans was unharmed by the lincosamide clindamycin and the oxazolidinone linezolid and grew at the maximal tested concentrations of these antibiotics, but these resistances could not be inferred from the ARG's. Moreover, $C$. metallidurans was sensitive to all fluoroquinolones and $\beta$-lactam antibiotics besides Penicillin $G$ in spite of possessing several efflux transporter genes from which a resistance against fluorquinolones can be inferred and also the $\beta$ lactamase AmpC which is a specialized cephalosporinase and infers resistance against the tested cefotaxime and ceftriaxone. However, it is known that these two cephalosporins, while being sensitive to AmpC, are only weak inducers for actual AmpC expression (60).

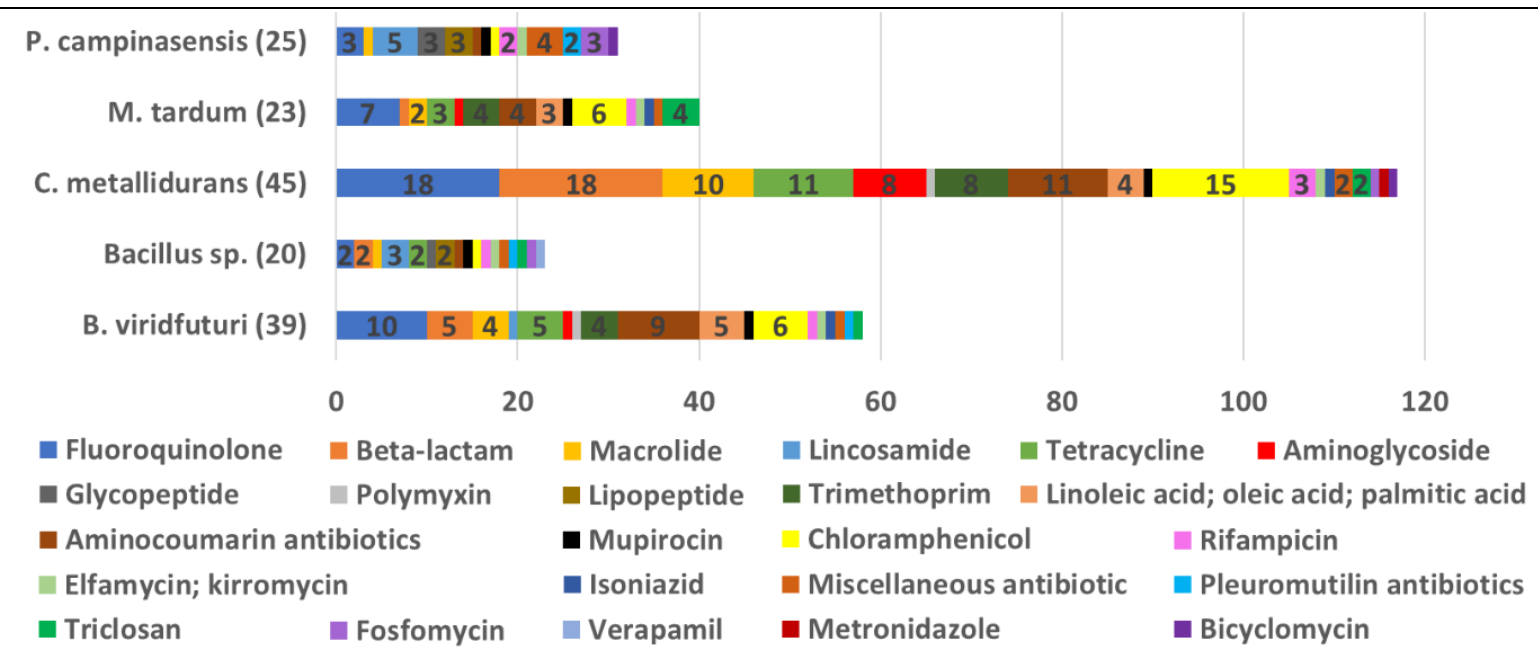

Fig. 10: Summary of the antibiotic resistance genes (ARG) detected in the sequenced genomes. The number in () is the total number of detected ARG in the respective genome and the colored columns show the groups of antibiotics against which the detected ARG are known to provide resistance. Numbers within a colored column indicate how many of the detected ARG provide resistance against this type of antibiotic; in case of one ARG was found, no number is given. As some ARG, especially the multidrug efflux pumps which were found in a high number in $C$. metallidurans and $B$. viridfuturi, may be able to provide resistance against multiple kinds of antibiotics, the sum of the numbers within the colored columns may exceed the total number of detected antibiotic resistance genes per organism. The number of ARG as well as of inferred antibiotic resistances was the same in the sequenced Bacillus genomes, which is why they are summarized as one Bacillus sp. in this graph.

The ISS microbiome in context: humans and the cleanroom as a contamination source 
clustered separately in multivariate analyses (Fig. 11). The cleanroom microbiome was specifically characterized by a predominant abundance of $\alpha$-Proteobacteria such as Novosphingobium, Sphingomonas and Methylobacterium whereas most ISS samples were dominated by Firmicutes and Actinobacteria. This is in accordance with previous findings (13). Based on this observation, we argue that the items delivered from terrestrial cleanrooms to the ISS are most likely not a (relevant) microbiome source.

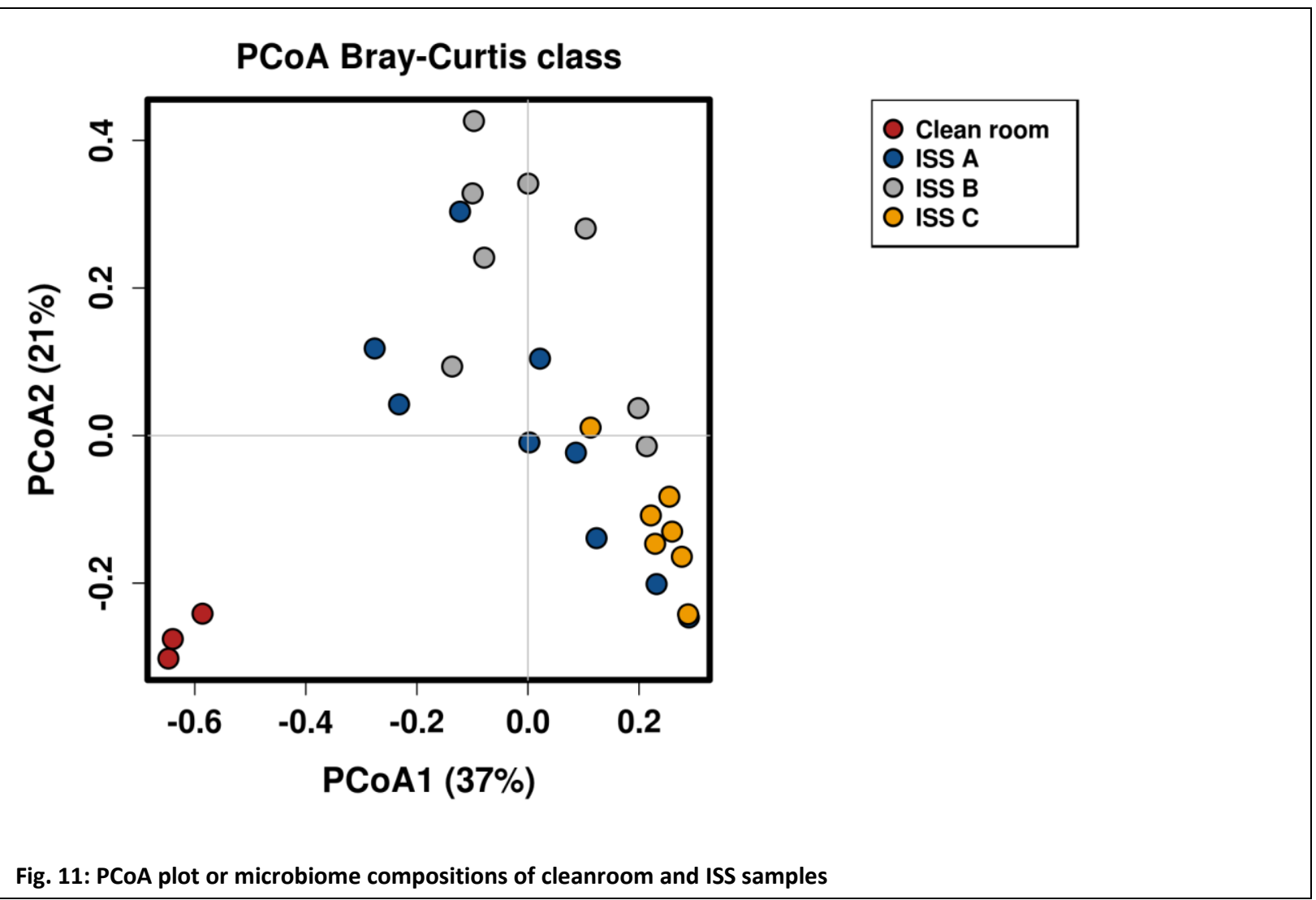

However, a more detailed picture was obtained, when we looked at the cultivable diversity retrieved from ISS and cleanroom samples (Fig. 12), where we found an overlap of several bacterial species, including: Bacillus cereus, B. aerophilus, B. subtilis, B. nealsonii, Micrococcus aloeverae, M. yunnanensis, Kocuria palustris, Ralstonia insidiosa. Human-associated microorganisms, such as e.g. Micrococcus species, were more likely introduced by humans into both environments than transported via cargo from cleanrooms to the ISS. Nevertheless, this comparison indicates a potential transfer of cleanroom associated microorganisms (such as Bacillus species) onto the ISS where they established themselves as a part of the ISS microbial community. Based on the total cultivable diversity they comprise only a minor fraction of the ISS microbial community. 


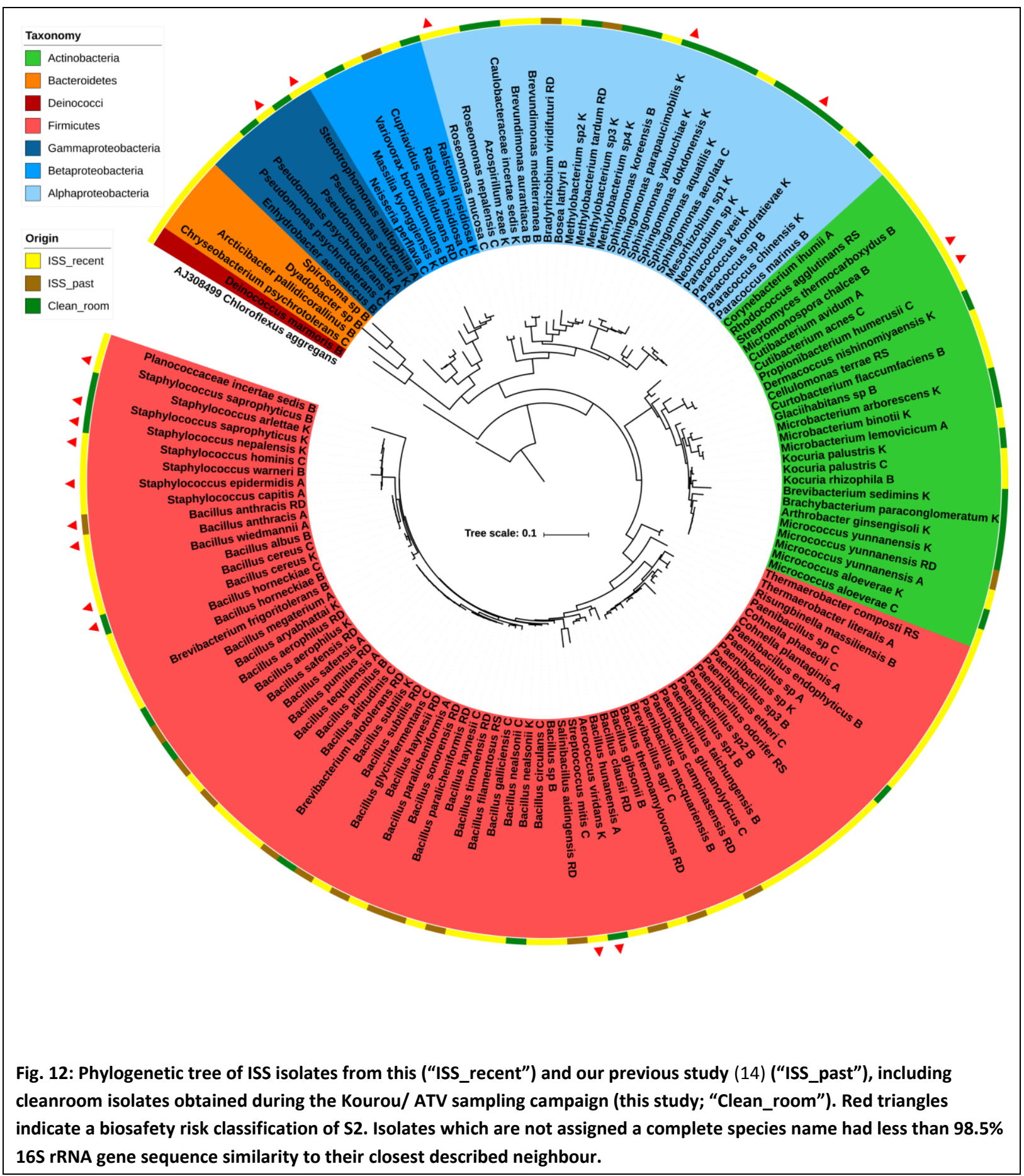

The application of 21 different cultivation approaches resulted in a high diversity of microbial isolates from the ISS, and 22 of the bacterial genera obtained during this study have not been isolated from ISS samples before, although most of them have been detected by molecular methods $(12,13,19)$ (Arcticibacter, Bosea, Brevundimonas, Chryseobacterium, Cohnella, Curtobacterium, Cutibacterium, 
Planococcus, Propionibacterium, Risungbinella, Roseomonas, Spirosoma, Stenotrophomonas, Thermaerobacter, and Variovorax). Of our fungal isolates, only Aspergillus unguis was not found on the ISS before.

Additionally eleven of the ISS isolates obtained in this study might even qualify to comprise novel, hitherto undescribed bacterial species as their 16S rRNA gene sequence similarity to their respective closest described neighbor was below $98.5 \%$. Six isolates thereof revealed a similarity lower than 97\%, including Paenibacillus, Dyadobacter, Spirosoma representatives. The isolate with the highest nucleotide difference was isolate R9_B3_IA sampled from the SSC laptop in the Columbus module, as closest neighbour was Planococcus halocryophilus (87.36\% sequence identity), an extreme psychrophile isolated from arctic permafrost (61).

\section{Do ISS microorganisms interact with ISS surfaces? Co-incubation experiments with ISS materials}

719 ISS isolates Cupriavidus metallidurans strain pH5_R2_1_IIA, Bacillus paralicheniformis strain 720 R2A_5R_0.5 (14) and Cutibacterium avidum strain R7A_A1_IIIA were aerobically and anaerobically 721 incubated together with untreated aluminium alloy platelets, eloxated aluminium alloy platelets, and pieces of NOMEX ${ }^{\circledR}$ fabric, to investigate if these isolates interact with these ISS surface materials. After incubation ( 6 weeks; $32^{\circ} \mathrm{C}$; liquid R2A medium), the co-incubated materials were analyzed via scanning electron microscopy (Fig. 13). The NOMEX ${ }^{\circledR}$ fabric itself remained intact over time (Fig. 13, IL, negative control), but served as an excellent attachment surface for B. paralicheniformis biofilms and single cells of $C$. metallidurans. The ability of $C$. metallidurans to attach to surfaces was found genetically encoded by pili formation capacity (see above). 

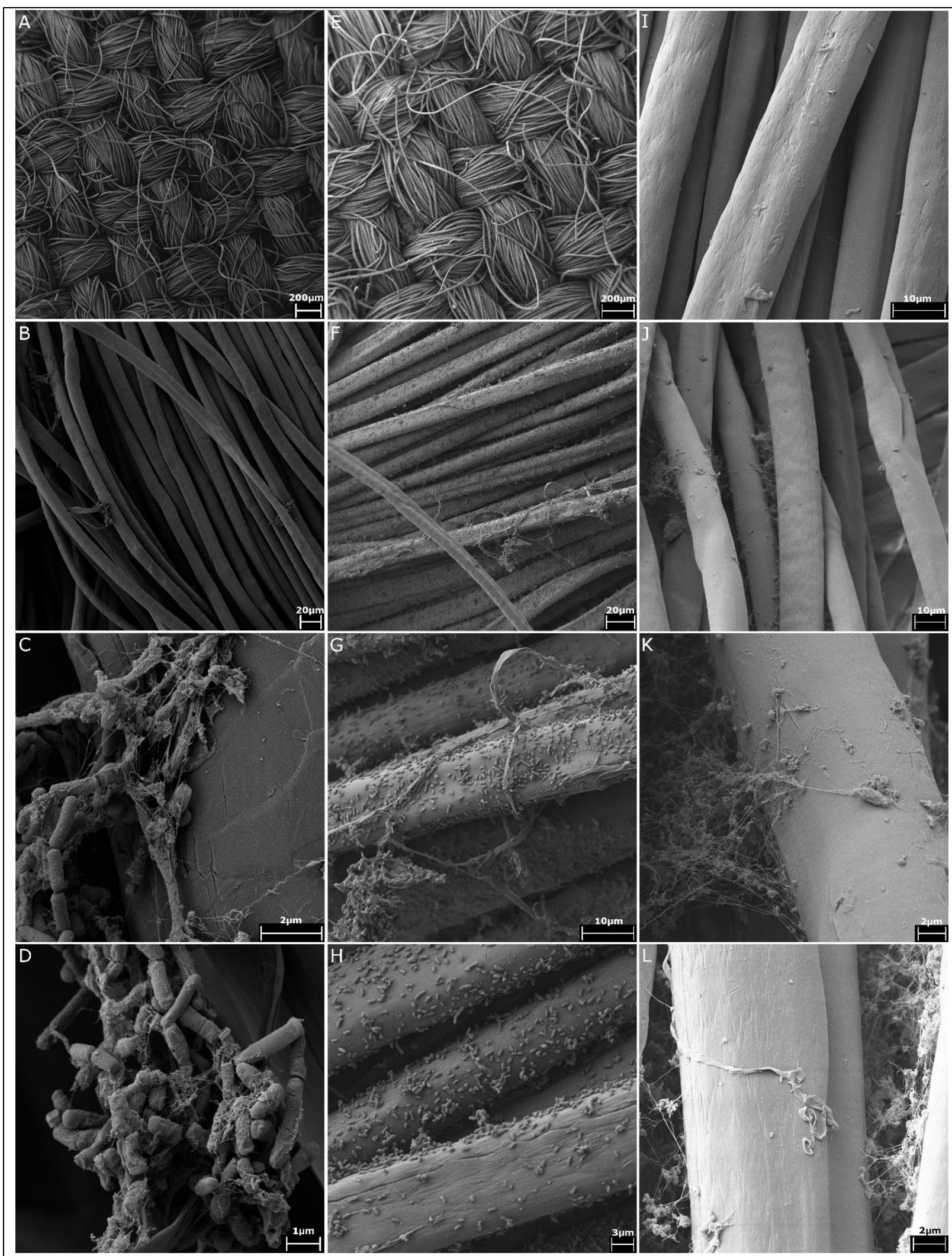

Fig. 13: Scanning electron micrographs of NOMEX ${ }^{\circledast}$ fabric which was co-incubated for six weeks with bacteria isolated from the ISS: A-D: Co-incubation with Bacillus paralicheniformis; E-H: Co-incubation with Cupriavidus metallidurans; I-L: Negative control of NOMEX ${ }^{\circledR}$ fabric kept in sterile medium for six weeks. Precipitates found in the negative control were found to be remnants of the medium/particles and did not contain microbial cells. 
735 B. paralicheniformis did neither adhere to the untreated nor to the eloxated aluminum alloy. 736 However, the untreated aluminum alloy which was co-incubated with B. paralicheniformis showed 737 sporadical signs of corrosion compared to the untreated negative control incubated in sterile 738 medium. All eloxated aluminum surfaces showed attached debris regardless if the incubation in the 739 sterile medium was performed under oxic or anoxic conditions. Single cells of $C$. metallidurans also 740 attached to the surfaces of untreated and eloxated aluminum alloys and their co-incubated 741 aluminum alloys had a unique background surface pattern, which was distinct from their respective 742 negative controls. Under anoxic conditions, $C$. avidum formed a biofilm attached to the surface of 743 both, untreated and eloxated, aluminum alloys. 
In this study, we aimed to exploit the microbial information obtained from three sampling events aboard the International Space Station with respect to: i) microbial sources, diversity and distribution within the ISS, ii) functional capacity of microbiome and microbial isolates, iii) extremotolerance and antibiotics-resistance (compared to ground controls), and iv) microbial behavior towards ISS-relevant materials (biofilm formation, potential degradation or corrosion).

Study limitations. Spaceflight experiments especially suffer from the limitations given by the circumstances that cannot be influenced by the scientists. This affected e.g. the number of samples to be taken (limited mass of payload), the sampling procedure (compatible to microgravity conditions and safety requirements), selection of sampling time points (according to assigned crew time and the overall mission planning), and the delivery duration of the samples to the laboratory. Being aware of these circumstances, experiments were planned accordingly ( $>5$ years implementation phase), and numerous controls were run to ensure the integrity of the information retrieved.

Microbiome sources and context. Based on our observations and previous reports (10), we confirm a mostly human-associated microbiome aboard the ISS (62). Other proposed sources are cargo delivery (due to the detected overlap of cleanroom/ cargo and ISS microbial community), food (such as seasoning, dried fruits, nuts and herbs, or even probiotics (63) (as indicated by the presence of e.g. Bacillus and Lactococcus signatures), and potentially the personal belongings brought to the ISS (possibly reflected by the increased diversity in the personal sleeping unit microbiome). It shall be noted, that cargo deliveries are cleaned or even disinfected before upload $(64,65)$, but an international standard for these procedures does not exist and thus the cleanliness of the cargo delivery might vary.

As a consequence, the ISS microbiome was characterized by a predominance of human (skin)associated Staphylococcus, Corynebacterium and Streptococcus signatures (66). In general, these microorganisms are typical indicators for confined indoor environments (cleanrooms, space stations, hospital areas such as intensive care unit, operating rooms; (10)), emphasizing the substantial contribution of the human microbiome (see also (13)). In more detail, all top 20 genera described in the hospital study (66) were also detected in the entire ISS microbiome (mostly also under top 20). Enhydrobacter (Pseudomonadales, a typical environmental species, (67)) was the only hospital top 20 genus which was not detected by the molecular approach in this study, but an Enhydrobacter aerosaccus isolate was obtained from the Columbus RGSH sample in session B.

778 Within the top 20 list of the ISS microbial signatures, Haemophilus, Aerococcus, Stenotrophomonas, 779 Gemella, Bacteroides, Actinomyces, Veillonella, Granulicatella, Blautia, Propionibacterium and 
Enterobacter could not be found in the top 20 hospital list, indicating that those were relatively higher abundant in the ISS or even specific for this location.

782 All of these genera are typical human-associated microorganisms and thrive in the oral/respiratory 783 tract (e.g. Haemophilus), on human skin (e.g. Propionibacterium) or the human gut (e.g. Blautia). Some of these microorganisms have opportunistic pathogenic potential, as also pointed out for Enterobacter species isolated from the ISS WHC (68). We obtained in total eleven ISS isolates belonging to biosafety risk group S2 during this study, including Pseudomonas putida isolated from the RGSH in Node2 and isolates of the Bacillus cereus/anthracis/thuringensis clade retrieved from the RGSHs in Node2 and Columbus, from the hand grips in Columbus, and from the sleeping unit in Node2. However, most of these are typical human-associated bacteria which have only opportunistic pathogenic potential. Especially in the light of a weakened human immune system in space conditions, the presence and abundance of such opportunistic pathogens has of course to be carefully monitored, but as these do thrive in and on the human body and are shed into the environment by the crew itself, such opportunistic pathogens will always exist in built environments and their presence per se is not alarming (10).

ISS microbiome diversity, biogeography and dynamics. The ISS microbiome was not found to be stable in composition and diversity, although a vast core microbiome existed over time and independent from location. However, specific microbial patterns could be identified for various functional areas within the station, as e.g. the WHC and RGSH showed the highest number of unique RSVs, and bacterial and in particular archaeal signatures were found to be specifically indicative for certain areas. This is in agreement with the observations of Ruiz-Calderon et al. that, increasing with the level of human interaction, indoor surfaces reflect space use and, besides, show increased content of human-associated microbial signatures (69).

Fluctuations in diversity and composition were detectable when two different time points (session A and B) were compared. It shall be mentioned, that no crew exchange took place during this period, but two cargo deliveries docked within this time frame (SpaceX and Soyuz), which could have influenced the microbiome composition. However, we detected an increase in specifically human (gut)-associated microorganisms (Escherichia/Shigella, Lachnoclostridium etc.) over the sampling period, of which the reason is unknown.

810 A different picture was obtained from the cultivable diversity of the ISS microbial community, with

811 Micrococcus yunnanensis, Bacillus hunanensis, B. megaterium and B. safensis and Staphylococcus 812 epidermidis being cultivable from both sampling sessions, representing hardy (spore-forming) and 813 human skin- associated microorganisms, whereas typical gut-associated microorganisms could not be 814 retrieved by our enrichments, as our cultivation approaches were designed to target rather 
environmental, extremotolerant microbes. Thus, the cultivation- and molecular-based microbial community analysis is not fully comparable due to the limitations in cultivation efforts.

Archaeal signatures were detected in 14 of the 24 ISS samples (universal and specific approach). Most earlier studies on the ISS microbiome ignored the possible presence of Archaea, but some of the more recent studies also reported the detection of Archaea aboard the ISS but did not further discuss their existence $(19,21)$. We found, that archaeal signatures were nicely reflecting the frequency of human contact and the type of surface (see Fig. 5). The detected sequences of Thaumarchaeota, Woesearchaeota and Methanobrevibacter (Euryarchaeota) have all been attributed to the human microbiome before (70-72).

\section{Functional capacity of microbiome and microbial isolates, extremo-tolerance and antibiotics-} resistance (compared to ground controls). In our study, we confirmed the pangenome-based observation of Blaustein et al. (24), on genomic, but also on isolate and resistance-pattern level that ISS microorganisms are not necessarily more extremophilic or antibiotic resistant than their groundrelatives with respect to growth behavior, or antibiotics resistances, nor their genomic inventory.

We rather argue that the ISS environment supports selection of the best-adapted microorganisms (e.g. spore-formers) towards the partially extreme physical and chemical environmental conditions (e.g. radiation, alkaline cleaning agents), but does not induce general changes in the physiological nor genomic capacities of microbes. Thus, were not able to confirm the null hypothesis that strains obtained from the ISS are more extremotolereant/extremophilic than closely related strains from Earth regarding the upper and lower boundaries of their temperature and $\mathrm{pH}$ growth ranges. With the exception of Bradyrhizobium viridifuturi pH5_R2_1_I_B, all tested ISS isolates were able to grow at $\mathrm{pH} 9$ or higher, which might be due to a selection pressure caused by alkaline cleaning reagents used on board the ISS.

Moreover, the data presented here show that the molecular detection of antibiotic resistance genes, while being a good approximation of the resistance potential of an organism or microbial community, does on the one hand overestimate the antibiotic resistance potential (as some resistance genes might not be expressed at all). On the other hand it does not necessarily cover all antibioticresistances which a microorganism actually has. Thus we advise coupling traditional cultivation approaches with molecular investigations to retrieve a full picture of the situation.

Microbial surface interaction with ISS materials. Although we could not confirm an increased threat of ISS microbiome towards crew's health, we observed that surface interaction is critical for the microbial community aboard. A variety of ISS surface materials are composed of metals, including alloy EN AW 2219 (aluminum copper magnesium), which might stress interaction with metal ions and 
849 settlement. In our co-incubation experiments, we could confirm that ISS microbial isolates can 850 adhere and grow on metal and in particular textile surfaces (NOMEX fabric), where local moisture 851 (e.g. condensate) could support biofouling, biofilm formation and material damage through acid 852 production $(6,73-75)$. This is particularly important with respect to fungal growth, as these might 853 affect human health indirectly by causing allergic reactions and asthmatic responses $(17,76-78)$.

\section{Conclusion: Microbes aboard: Reason for concern?}

855 Although we cannot fully exclude a threat of the ISS microbiome towards crew health (in particular in 856 interaction with the weakened human immune system) our data do not indicate direct reason for 857 concern. However, we raise special attention to the microbial-surface interaction problem in order to 858 avoid biofouling and biofilm formation, which could directly impact material integrity and indirectly 859 human health and therefore pose a potential risk to mission success. 


\section{Acknowledgements.}

863

864

865

866

867

868

869

870

871

872

873

874

875

876

877

878

879

880

881

882

883

884

885

886

887

888

889

890

891

892

893

894

895

896

897

898

899

900

901

902

We are thankful for financial support by the FFG (Austrian Research Promotion Agency, Project No. 847977) and the European Space Agency (ESA) for financing and realization of this space project as part of the ELIPS program (ILSRA-2009-1053 ARBEX). We are very grateful for the scientific and technical support by Lobke Zuijderduijn, Stefanie Raffestin (sampling in Kourou), the BIOTESC team (Lucerne University of Applied Sciences), the ISS crew and all other involved members of space agencies and attached institutes. We thank Elisabeth Grohmann for providing a Staphylococcus strain isolated from the Concordia station. PhD student Maximilian Mora was supported by the local PhD program MolMed.

\section{References}

1. Sonnenfeld G, Shearer WT. Immune function during space flight. Nutrition [Internet]. 2002;18(10):899-903.

2. Aponte VM, Finch DS, Klaus DM. Considerations for non-invasive in-flight monitoring of astronaut immune status with potential use of MEMS and NEMS devices. Life Sci [Internet]. 2006;79(14):1317-33.

3. Wilson N. A Microbial Hitchhiker's Guide to the GalaxyResearchers race to understand effects of deep space on microbiome. Bioscience. 2019 Jan;69(1):5-11.

4. Wilson JW, Ott CM, zu Bentrup KH, Ramamurthy R, Quick L, Porwollik S, et al. Space flight alters bacterial gene expression and virulence and reveals a role for global regulator $\mathrm{Hfq}$. Proc Natl Acad Sci. 2007;104(41):16299-304.

5. Hammond TG, Stodieck L, Birdsall HH, Becker JL, Koenig P, Hammond JS, et al. Effects of Microgravity on the Virulence of Listeria monocytogenes, Enterococcus faecalis, Candida albicans, and Methicillin-Resistant Staphylococcus aureus. Astrobiology. 2013;13(11):108190 .

6. Alekhova TA, Aleksandrova AA, Novozhilova TY, Lysak L V, Zagustina NA, Bezborodov AM. Monitoring of Microbial Degraders in Manned Space Stations. Appl Biochem Microbiol [Internet]. 2005;41(4):382-9.

7. Novikova ND, Polikarpov NA, Poddubko S V, Deshevaya EA. The results of microbiological research of environmental microflora of orbital station Mir. 2001.

8. Novikova ND. Review of the knowledge of microbial contamination of the Russian manned spacecraft. Microb Ecol. 2004;47(2):127-32.

9. Schwendner P, Mahnert A, Koskinen K, Moissl-Eichinger C, Barczyk S, Wirth R, et al. Preparing for the crewed Mars journey: microbiota dynamics in the confined Mars500 habitat during simulated Mars flight and landing. Microbiome. 2017;5(1):129.

10. Mora M, Mahnert A, Koskinen K, Pausan MR, Oberauner-Wappis L, Krause R, et al. Microorganisms in confined habitats: Microbial monitoring and control of the International Space Station, cleanrooms, operating rooms and intensive care units. Front Microbiol. 2016;7.

11. Coil DA, Neches RY, Lang JM, Brown WE, Severance M, Cavalier DD, et al. Growth of 48 built environment bacterial isolates on board the International Space Station (ISS). 2016.

12. Venkateswaran K, Vaishampayan P, Cisneros J, Pierson DL, Rogers SO, Perry J. International 
Space Station environmental microbiome - Microbial inventories of ISS filter debris. Appl Microbiol Biotechnol. 2014;98(14):6453-66.

13. Checinska A, Probst AJ, Vaishampayan P, White JR, Kumar D, Stepanov VG, et al. Microbiomes of the dust particles collected from the International Space Station and Spacecraft Assembly Facilities. Microbiome [Internet]. 2015;3(1):1-18.

14. Mora M, Perras AK, Alekhova TA, Wink L, Krause R, Aleksandrova A, et al. Resilient microorganisms in dust samples of the International Space Station - survival of the adaptation specialists. Microbiome. 2016;4(1):65.

15. Nicholas AB, Avila-Herrera A, Allen JE, Singh N, Sielaff AC, Jaing C, et al. Whole metagenome profiles of particulates collected from the International Space Station. Microbiome. 2017;5(1):81.

16. Voorhies AA, Lorenzi HA. The challenge of maintaining a healthy microbiome during longduration space missions. Front Astron Sp Sci. 2016;3:23.

17. Romsdahl J, Blachowicz A, Chiang AJ, Singh N, Stajich JE, Kalkum M, et al. Characterization of Aspergillus niger Isolated from the International Space Station. mSystems. 2018;3(5):e001128.

18. Seuylemezian A, Vaishampayan P, Cooper K, Venkateswaran K. Draft Genome Sequences of Acinetobacter and Bacillus Strains Isolated from Spacecraft-Associated Surfaces. Genome Announc. 2018;6(6):e01554-17.

19. Lang JM, Coil DA, Neches RY, Brown WE, Cavalier D, Severance M, et al. A microbial survey of the International Space Station (ISS). PeerJ. 2017;5:e4029.

20. Urbaniak C, Sielaff AC, Frey KG, Allen JE, Singh N, Jaing C, et al. Detection of antimicrobial resistance genes associated with the International Space Station environmental surfaces. Sci Rep. 2018;8(1):814.

21. Singh NK, Wood JM, Karouia F, Venkateswaran K. Succession and persistence of microbial communities and antimicrobial resistance genes associated with International Space Station environmental surfaces. Microbiome. 2018;6(1):204.

22. Crucian B, Babiak-Vazquez A, Johnston S, Pierson DL, Ott CM, Sams C. Incidence of clinical symptoms during long-duration orbital spaceflight. Int J Gen Med. 2016;9:383.

23. Van Houdt R, Mijnendonckx K, Leys N. Microbial contamination monitoring and control during human space missions. Planet Space Sci. 2012;60(1):115-20.

24. Blaustein RA, McFarland AG, Maamar S Ben, Lopez A, Castro-Wallace S, Hartmann EM. Pangenomic Approach To Understanding Microbial Adaptations within a Model Built Environment, the International Space Station, Relative to Human Hosts and Soil. mSystems. 2019;4(1):e00281-18.

25. Watve M, Shejval V, Sonawane C, Rahalkar M, Matapurkar A, Shouche Y, et al. The'K'selected oligophilic bacteria: A key to uncultured diversity? Curr Sci. 2000;1535-42.

26. StiegImeier M, Wirth R, Kminek G, Moissl-Eichinger C. Cultivation of anaerobic and facultatively anaerobic bacteria from spacecraft-associated clean rooms. Appl Environ Microbiol. 2009;75(11):3484-91.

27. Balch WE, Fox GE, Magrum LJ, Woese CR, Wolfe RS. Methanogens: reevaluation of a unique 
biological group. Microbiol Rev. 1979;43(2):260.

28. Sauder LA, Albertsen M, Engel K, Schwarz J, Nielsen PH, Wagner M, et al. Cultivation and characterization of Candidatus Nitrosocosmicus exaquare, an ammonia-oxidizing archaeon from a municipal wastewater treatment system. ISME J. 2017;11(5):1142.

29. StiegImeier M, Rettberg P, Barczyk S, Bohmeier M, Pukall R, Wirth R, et al. Abundance and diversity of microbial inhabitants in European spacecraft-associated clean rooms. Astrobiology [Internet]. 2012;12(6):572-85.

30. Yoon SH, Ha SM, Kwon S, Lim J, Kim Y, Seo H, et al. Introducing EzBioCloud: A taxonomically united database of $16 \mathrm{~S}$ rRNA gene sequences and whole-genome assemblies. Int J Syst Evol Microbiol. 2017;67(5):1613-7.

31. Kõljalg U, Nilsson RH, Abarenkov K, Tedersoo L, Taylor AFS, Bahram M. Towards a unified paradigm for sequence-based identification of fungi. Mol Ecol. 2014;22(November 2013):5271-7.

32. Ratnasingham S, Hebert PDN. BARCODING, BOLD : The Barcode of Life Data System (www.barcodinglife.org). Mol Ecol Notes. 2007;7(April 2016):355-64.

33. Price MN, Dehal PS, Arkin AP. FastTree 2-approximately maximum-likelihood trees for large alignments. PLoS One. 2010;5(3):e9490.

34. Letunic I, Bork P. Interactive Tree Of Life (iTOL): an online tool for phylogenetic tree display and annotation. Bioinformatics. 2006/10/20 ed. 2007;23(1):127-8.

35. Moissl-Eichinger C. Archaea in artificial environments: their presence in global spacecraft clean rooms and impact on planetary protection. ISME J [Internet]. 2011;5(2):209-19.

36. Caporaso JG, Lauber CL, Walters WA, Berg-Lyons D, Huntley J, Fierer N, et al. Ultra-highthroughput microbial community analysis on the Illumina HiSeq and MiSeq platforms. ISME J. 2012;6(8):1621-4.

37. Probst AJ, Auerbach AK, Moissl-Eichinger C. Archaea on human skin. PLoS One. 2013;8(6):e65388.

38. Raskin L, Stromley JM, Rittmann BE, Stahl DA. Group-specific 16S rRNA hybridization probes to describe natural communities of methanogens. Appl Environ Microbiol. 1994 Apr;60(4):123240.

39. Stahl DA, Amann R. Development and Application of Nucleic Acid Probes in Bacterial Systematics. In: Stackebrandt E, Goodfellow M, editors. Nucleic Acid Techniques in Bacterial Systematics. 1991. p. 205-48.

40. Klindworth A, Pruesse E, Schweer T, Peplies J, Quast C, Horn M, et al. Evaluation of general $16 \mathrm{~S}$ ribosomal RNA gene PCR primers for classical and next-generation sequencing-based diversity studies. Nucleic Acids Res. 2012;gks808.

41. Mora M, Perras A, Alekhova TA, Wink L, Krause R, Aleksandrova A, et al. Resilient microorganisms in dust samples of the International Space Station-survival of the adaptation specialists. Microbiome. 2016;4(1).

42. Andrews S. FastQC: a quality control tool for high throughput sequence data. 2010.

43. Bolger AM, Lohse M, Usadel B. Trimmomatic: A flexible trimmer for Illumina sequence data. 
Bioinformatics. 2014;30(15):2114-20.

44. Bankevich A, Nurk S, Antipov D, Gurevich AA, Dvorkin M, Kulikov AS, et al. SPAdes: A New Genome Assembly Algorithm and Its Applications to Single-Cell Sequencing. J Comput Biol. 2012;19(5):455-77.

45. Parks DH, Imelfort M, Skennerton CT, Hugenholtz P, Tyson GW. CheckM: assessing the quality of microbial genomes recovered from. Cold Spring Harb Lab Press Method. 2015;1:1-31.

46. Vallenet D, Calteau A, Cruveiller S, Gachet M, Lajus A, Josso A, et al. MicroScope in 2017: An expanding and evolving integrated resource for community expertise of microbial genomes. Nucleic Acids Res. 2017;45(D1):D517-28.

47. Vallenet D, Labarre L, Rouy Z, Barbe V, Bocs S, Cruveiller S, et al. MaGe: A microbial genome annotation system supported by synteny results. Nucleic Acids Res. 2006;34(1):53-65.

48. Vallenet D, Engelen S, Mornico D, Cruveiller S, Fleury L, Lajus A, et al. MicroScope: A platform for microbial genome annotation and comparative genomics. Database. 2009;2009:1-12.

49. ECSS EC for SS. Microbial examination of flight hardware and cleanrooms ECSS-Q-ST-70-55C. 2008;

50. Eucast. PK/PD (Non-species related) breakpoints [Internet]. [cited 2016 Jul 1].

51. Callahan BJ, McMurdie PJ, Rosen MJ, Han AW, Johnson AJA, Holmes SP. DADA2: Highresolution sample inference from Illumina amplicon data. Nat Methods. 2016;13:581-3.

52. Callahan BJ, Sankaran K, Fukuyama JA, McMurdie PJ, Holmes SP. Bioconductor workflow for microbiome data analysis: from raw reads to community analyses. F1000Research. 2016;5.

53. Zakrzewski M, Proietti C, Ellis JJ, Hasan S, Brion M-J, Berger B, et al. Calypso: a user-friendly web-server for mining and visualizing microbiome-environment interactions. Bioinformatics. 2016 Dec;btw725.

54. Aßhauer KP, Wemheuer B, Daniel R, Meinicke P. Tax4Fun: predicting functional profiles from metagenomic 16S rRNA data. Bioinformatics. 2015;31(17):2882-4.

55. Caporaso JG, Kuczynski J, Stombaugh J, Bittinger K, Bushman FD, Costello EK, et al. QIIME allows analysis of high-throughput community sequencing data. Nat Methods. 2010;7(5):3356.

56. Shannon P, Markiel A, Ozier O, Baliga NS, Wang JT, Ramage D, et al. Cytoscape: a software environment for integrated models of biomolecular interaction networks. Genome Res. 2003;13(11):2498-504.

57. Thannesberger J, Hellinger H-J, Klymiuk I, Kastner M-T, Rieder FJJ, Schneider M, et al. Viruses comprise an extensive pool of mobile genetic elements in eukaryote cell cultures and human clinical samples. FASEB J. 2017;31(5):1987-2000.

58. Meyer F, Paarmann D, D'Souza M, Olson R, Glass EM, Kubal M, et al. The metagenomics RAST server-a public resource for the automatic phylogenetic and functional analysis of metagenomes. BMC Bioinformatics. 2008;9(1):386.

59. Bolyen E, Rideout JR, Dillon MR, Bokulich NA, Abnet C, Al-Ghalith GA, et al. QIIME 2: Reproducible, interactive, scalable, and extensible microbiome data science. 2018. 
Contents.

61. Mykytczuk NCS, Wilhelm RC, Whyte LG. Planococcus halocryophilus sp. nov., an extreme subzero species from high Arctic permafrost. Int J Syst Evol Microbiol. 2012;62(Pt 8):1937-44.

62. Yamaguchi N, Ichijo T, Nasu M. Bacterial Monitoring in the International Space Station\&ldquo;Kibo\&rdquo; Based on rRNA Gene Sequence. Trans JAPAN Soc Aeronaut Sp Sci Aerosp Technol JAPAN. 2016;14(ists30):Pp_1 - Pp_4.

63. Sakai T, Moteki Y, Takahashi T, Shida K, Kiwaki M, Shimakawa Y, et al. Probiotics into outer space: feasibility assessments of encapsulated freeze-dried probiotics during 1 month's storage on the International Space Station. Sci Rep. 2018;8(1):10687.

64. Pierson DL. Microbial contamination of spacecraft. Gravit Sp Biol Bull. 2001;14:1-6.

65. Mord ISS. SSP 50260: ISS medical operations requirement document. Houston. 2009;307:22.

66. Lax S, Sangwan N, Smith D, Larsen P, Handley KM, Richardson M, et al. Bacterial colonization and succession in a newly opened hospital. Sci Transl Med. 2017;9(391):eaah6500.

67. Staley JT, Irgens RL, Brenner DJ. Enhydrobacter aerosaccus gen. nov., sp. nov., a gasvacuolated, facultatively anaerobic, heterotrophic rod. Int J Syst Evol Microbiol. 1987;37(3):289-91.

68. Singh NK, Bezdan D, Sielaff AC, Wheeler K, Mason CE, Venkateswaran K. Multi-drug resistant Enterobacter bugandensis species isolated from the International Space Station and comparative genomic analyses with human pathogenic strains. BMC Microbiol. 2018;18(1):175.

69. Ruiz-Calderon JF, Cavallin H, Song SJ, Novoselac A, Pericchi LR, Hernandez JN, et al. Walls talk: Microbial biogeography of homes spanning urbanization. Sci Adv. 2016;2(2):e1501061.

70. Moissl-Eichinger C, Probst AJ, Birarda G, Auerbach A, Koskinen K, Wolf P, et al. Human age and skin physiology shape diversity and abundance of Archaea on skin. Sci Rep. 2017;7 (1):4039.

71. Koskinen K, Pausan MR, Perras AK, Beck M, Bang C, Mora M, et al. First insights into the diverse human archaeome: Specific detection of Archaea in the gastrointestinal tract, lung, and nose and on skin. MBio. 2017;8(6).

72. Pausan MR, Csorba C, Singer G, Till H, Schoepf V, Santigli E, et al. Measuring the archaeome: detection and quantification of archaea signatures in the human body. bioRxiv [Internet]. 2018 Jan 1;

73. Alekhova TA, Zagustina NA, Aleksandrova A V, Novozhilova TY, Borisov A V, Plotnikov AD. Monitoring of initial stages of the biodamage of construction materials used in aerospace equipment using electron microscopy. J Surf Investig X-ray, Synchrotron Neutron Tech. 2007;1(4):411-6.

74. Alekhova TA, Shklover VY, Zagustina NA, Shvyndina N V, Plotnikov AD, Vasil'ev AL. Electron microscopy investigation of AIMg6 aluminum alloy surface defects caused by microorganisms extracted in space stations. J Surf Investig X-ray, Synchrotron Neutron Tech. 2010;4(5):74753.

75. Alekhova TA, Aleksandrova A V, Novozhilova TY, Lysak L V, Zagustina NA. The experiment "initial stages of biological damage and deterioration in space." Moscow Univ Biol Sci Bull. 2008;63(4):163-9. 
76. Baxi SN, Portnoy JM, Larenas-Linnemann D, Phipatanakul W, Barnes C, Baxi S, et al. Exposure and health effects of fungi on humans. J Allergy Clin Immunol Pract. 2016;4(3):396-404.

77. Blachowicz A, Chiang AJ, Romsdahl J, Kalkum M, Wang CCC, Venkateswaran K. Proteomic characterization of Aspergillus fumigatus isolated from air and surfaces of the International Space Station. Fungal Genet Biol. 2019;

78. Satoh K, Yamazaki T, Nakayama T, Umeda Y, Alshahni MM, Makimura M, et al. Characterization of fungi isolated from the equipment used in the International Space Station or Space Shuttle. Microbiol Immunol. 2016;60(5):295-302. 